Research Article

\title{
Research on the Obstruction Process of Rigid Netting Barriers toward Granular Flow
}

\author{
Yunyun Fan $\mathbb{D i D}^{1}$ and Fengyuan $\mathrm{Wu}^{2}$ \\ ${ }^{1}$ Key Laboratory of Ministry of Education on Safe Mining of Deep Metal Mines, Northeastern University, \\ Shenyang 110004, China \\ ${ }^{2}$ School of Civil Engineering, Shenyang Jianzhu University, Shenyang 110168, China
}

Correspondence should be addressed to Yunyun Fan; yunyunfan@126.com

Received 11 February 2019; Revised 29 April 2019; Accepted 8 May 2019; Published 19 June 2019

Academic Editor: Sanjay Nimbalkar

Copyright (c) 2019 Yunyun Fan and Fengyuan Wu. This is an open access article distributed under the Creative Commons Attribution License, which permits unrestricted use, distribution, and reproduction in any medium, provided the original work is properly cited.

\begin{abstract}
With the advantages of a simple structure and rapid construction, the rigid netting barrier (RNB) exerts a good obstruction effect on granular flow and is a common engineering measure used to prevent geological disasters in the form of granular flows. However, due to the limitations of current measuring and testing techniques, it is difficult to obtain an accurate measurement of the granular flow velocity and the impact force of granular flow on the mesh structures that are of primary concern in the design of protective structures. To study the characteristics of the obstruction process of RNBs toward granular flow, a typical impact experiment involving granular flow was numerically simulated by the discrete element method, and the correctness and effectiveness of the calculation method were also verified. On this basis, the discrete element method was applied to simulate the obstruction process affecting granular flow under different RNB setting conditions, and the calculation results clearly present the phenomena that occur during the obstruction process of RNBs toward granular flow, such as "run-up," "overflow," "passingthrough," and "grain-size segregation." By analyzing the effects of these phenomena on the obstruction efficiency and the time history of the forces acting on the RNB, the rational setting of an RNB was further discussed. This study can provide a reference for the engineering application of RNB.
\end{abstract}

\section{Introduction}

There are many physical phenomena included in granular flows with highly mobile natures, such as avalanches, landslides, rockfalls, and debris flows, which cause human casualties and damage to infrastructure [1-3]. Over the years, various engineering measures have been used to mitigate or prevent natural granular flow disasters. Common prevention and control measures generally include civil engineering works such as check dams, grid-type dams, lateral walls, deflection and terminal walls, and berms or barriers that control debris flows $[4,5]$. However, if the prevention and control engineering measures fail due to unreasonable engineering layout or insufficient project argument, they may cause more serious damage and loss $[6,7]$. Therefore, it is necessary to study in depth the impact of granular flows, the obstruction effect of barriers, and the structural design and rational layout of prevention and control measures.

The barriers are commonly used as control measures in engineering to reduce the mobility of granular flow and slow the granular flow movement $[8,9]$. Furthermore, the interaction between the grains and the structure can promote energy dissipation from the granular flow [10]. The barriers are often designed using empirical methods, and existing research on the interaction mechanism between granular flows and barriers is not deep enough [11]. Therefore, the study of the interaction between granular materials and the barriers under different barrier setting conditions is of great significance for more effective control structure design [12-14].

In recent years, several large-scale experiments [15] have been carried out to monitor the impact of granular flows 
with different material properties on barriers under different environmental conditions [16-18]. These research results provide valuable basic data and a solid foundation for the study of the obstruction process of barriers toward granular flows. However, due to the limitations of measuring and testing methods, field investigations and large-scale testing cannot clarify the complex interaction mechanism between granular flows and barriers [19]. Flume experiments indicate that the barriers can greatly reduce the flow velocity [20]. This reduction can be estimated empirically on the basis of the cross-sectional blockage over the channel area, but it only takes into account geometric effects, without any consideration of the interaction between granular flows and barriers [21]. Then, the dynamic behavior of dry granular flows toward barriers has been further studied $[9,13,22-24]$. In the studies, some more critical influence mechanisms were revealed, such as the formation of dead zones [21, 25] and run-ups $[26,27]$. The dead zones allow momentum to run-up vertically along the barrier [28], and the run-ups reduce the load on the top of the barrier and increase the impact pressure. The granular material gradually accumulates at the base of the barriers and rapidly attenuates energy by shearing of the grains to suppress the run-up height against the barriers. Further studies have shown that grain size and gradation both exert a great influence on the impact process, thus resulting in larger run-up heights and peak impact forces $[29,30]$.

With the rapid development of scientific computing technologies and modern mechanics theories, numerical simulations have become an important means to study the interaction process between granular flows and barriers. So far, the continuous model used for granular flow simulation is primarily based on the depth-averaged theory $[21,29,31-34]$ and is similar to the shallow water model. The main differences of the continuous model are that different bottom resistance terms were used for the corresponding granular material types $[2,29,33]$ and that the lateral pressure coefficient related to the motion state was introduced [21, 29, 31, 35]. Although the continuous model has been widely applied in large-scale granular flow simulations using different numerical methods, the depthaveraged theory is essentially a planar two-dimensional model, which can neither yield more information about the mechanical behavior of the granular material against structures [36,37] nor supply the concerned barrier peak impact forces when applied in the simulation of granular flows with vertical momentum $[30,38]$. Therefore, the discrete element method is more suitable for simulations of the physical process of granular flows impacting barriers $[24,39-42]$. Numerical simulation by the discrete element method can reproduce the processes of experiments or actual disasters, and the simulation results can be verified by comparison with experimental results. Furthermore, the complex interactions between granular flows and barriers and the main pathways of energy dissipation can also be better understood through analysis $[10,11]$. However, the discrete element method has higher requirements for calculation, so it is only appropriate for use to analyze the interaction between small-scale granular flows and barriers and to study the corresponding mechanisms [14].

Obviously, the current limited understanding of the obstruction process of barriers toward granular flow has hindered the development of protective barrier designs [43]. This study focused on the widely used rigid netting barriers with a simple structure (for convenience in the text, the rigid netting barrier is abbreviated as RNB). The discrete element method was used to simulate the obstruction process of RNB toward granular flow under different setting conditions. At the same time, the mechanisms of some special phenomena, the impact of granular flow on barriers, and the deceleration and obstruction effects exerted on granular flow by barriers were also studied. Furthermore, the numerical simulation can compensate for the insufficiency of the current test technology in measuring granular flow velocity and its impact on the RNB. Finally, the discrete element method can provide suggestions for the rational design and optimization of barrier layouts.

\section{Numerical Methods}

The software PFC3D based on the discrete element method was used for numerical calculations. According to the characteristics of this study, the linear contact model and the slip model were selected, and quartz sand was simulated by clumps.

2.1. Contact Model. In the contact-stiffness models shown in Figure 1, the force between two entities in contact is decomposed into normal force and shear force [44].

The normal force and the increment of the shear force can be, respectively, expressed as follows:

$$
\begin{aligned}
F_{i}^{n} & =K^{n} U^{n} n_{i}, \\
\Delta F_{i}^{s} & =K^{s} \Delta U_{i}^{s},
\end{aligned}
$$

where $F_{i}^{n}$ is the normal force, $K^{n}$ is the normal stiffness at the contact, $U^{n}$ is the normal overlap of discrete elements, $n_{i}$ is the unit normal vector, $\Delta F_{i}^{s}$ is the increment of shear force, $K^{s}$ is the shear stiffness at the contact, and $\Delta U_{i}^{s}$ is the relative displacement increment in the shear direction.

It can be seen from Equation (1) that the relationship between the contact force and the relative displacement can be expressed by the contact stiffness, including the normal stiffness and shear stiffness. The linear contact model is the most commonly used contact model, which assumes that the stiffness is a continuous variable.

The normal contact stiffness and the shear contact stiffness between contact entities A and B (which could be a ball, a clump, a wall, a line wall, or other elements) are, respectively, expressed as follows:

$$
\begin{aligned}
K^{n} & =\frac{k_{n}^{[\mathrm{A}]} k_{n}^{[\mathrm{B}]}}{k_{n}^{[\mathrm{A}]}+k_{n}^{[\mathrm{B}]}}, \\
K^{n} & =\frac{k_{s}^{[\mathrm{A}]} k_{s}^{[\mathrm{B}]}}{k_{s}^{[\mathrm{A}]}+k_{s}^{[\mathrm{B}]}},
\end{aligned}
$$




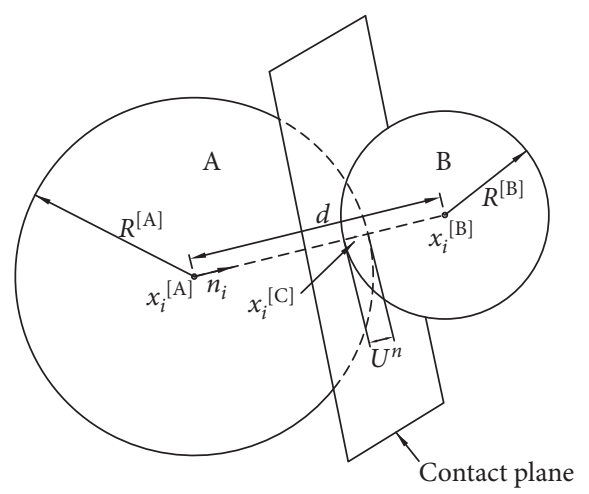

(a)

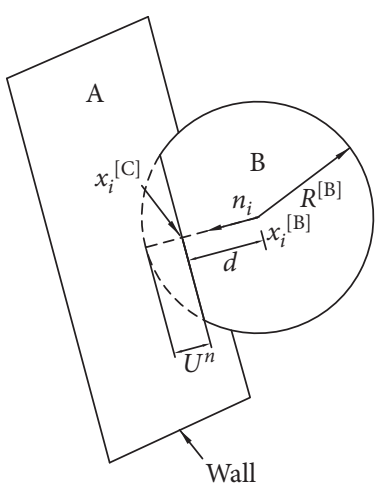

(b)

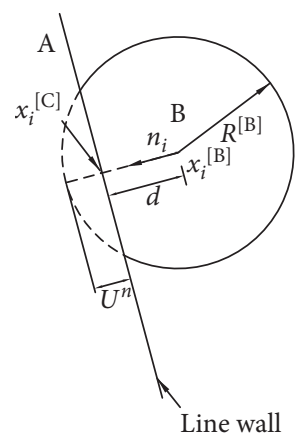

(c)

FIGURE 1: Sketch of the contact: (a) the contact between balls; (b) the contact between a ball and a wall; (c) the contact between a ball and a line wall.

where $k_{n}$ and $k_{s}$ represent the normal stiffness and shear stiffness of the two entities, respectively.

Furthermore, when the absolute value of the shear force at the contact $\left|F_{i}^{s}\right|$ is greater than the maximum allowable shear contact force $F_{\max }^{s}=\mu\left|F_{i}^{n}\right|$ (where the friction coefficient at the contact $\mu$ is equal to the minimum of the friction coefficient of the two contact entities), relative sliding occurs between the entities, and $F_{i}^{s}=\operatorname{sgn}\left(F_{i}^{s}\right) F_{\max }^{s}$.

2.2. Equations of Motion for a Clump. To simulate discrete elements with complex shapes, balls can be clumped together. The clump can generally be regarded as a rigid body and allows the existence of overlap between the balls. The contact force inside the clump was not taken into account in the calculation, and only the external contact force was considered [44].

In addition to the total mass $m$ and the location of the centroid $x_{i}^{[\mathrm{G}]}$, the basic properties of a clump model are the inertia moment $I_{i i}$ and the inertia products $I_{i j}$. These properties can be expressed in the following equation:

$$
\begin{aligned}
I_{i i} & =\sum_{p=1}^{N_{p}}\left\{m^{[\mathrm{p}]}\left(x_{j}^{[\mathrm{p}]}-x_{j}^{[\mathrm{G}]}\right)\left(x_{j}^{[\mathrm{p}]}-x_{j}^{[\mathrm{G}]}\right)+\frac{\left(2 m^{[\mathrm{p}]} R^{[\mathrm{p}]} R^{[\mathrm{p}]}\right)}{5}\right\}, \\
I_{i j} & =\sum_{p=1}^{N_{p}}\left\{m^{[\mathrm{p}]}\left(x_{i}^{[\mathrm{p}]}-x_{i}^{[\mathrm{G}]}\right)\left(x_{j}^{[\mathrm{p}]}-x_{j}^{[\mathrm{G}]}\right)\right\}, \quad j \neq i .
\end{aligned}
$$

The clump model moves like a rigid body. The centroid moment $M_{i}$ can be calculated as follows:

$$
M_{i}=\sum_{p=1}^{N_{p}}\left(\tilde{M}_{i}^{[\mathrm{p}]}+\varepsilon_{i j k}\left(x_{j}^{[\mathrm{p}]}-x_{j}^{[\mathrm{G}]}\right) F_{k}^{[\mathrm{p}]}+\sum_{c=1}^{N_{c}} \varepsilon_{i j k}\left(x_{j}^{[\mathrm{c}]}-x_{j}^{[\mathrm{p}]}\right) F_{k}^{[\mathrm{p}, \mathrm{c}]}\right)
$$

where the centroid moment $\widetilde{M}_{i}^{[\mathrm{p}]}$ is the applied moment of the grain, $F_{k}^{[p]}$ is the resultant force acting on the grain, and $F_{k}^{[\mathrm{p}, \mathrm{c}]}$ is the contact force acting on the grain.

The rate of change of the angular momentum $\dot{H}_{i}$ can be calculated as follows:

$$
\dot{H}_{i}=\dot{\omega}_{i} I_{i i}+\dot{\omega}_{j} I_{i j}+\varepsilon_{i j k} \omega_{j}\left(\omega_{k} I_{k k}-\omega_{l} I_{k l}\right), \quad j \neq i, l \neq k .
$$

Since $M_{i}=\dot{H}_{i}$, the rotational motion of a clump can be found by simultaneously solving Equations (4) and (5).

The equation for translational motion can be written in the following form:

$$
F_{i}=\sum_{p=1}^{N_{p}}\left(\widetilde{F}_{i}^{[\mathrm{p}]}+\sum_{c=1}^{N_{c}} F_{i}^{[\mathrm{p}, \mathrm{c}]}\right)=m\left(\ddot{x}_{i}-g_{i}\right)
$$

where $F_{i}$ is the resultant force, $\widetilde{F}_{i}^{[\mathrm{p}]}$ is the externally applied force acting on the grain, $F_{i}^{[\mathrm{p}, \mathrm{c}]}$ is the force acting on the grain at the contact, and $g_{i}$ is the body force acceleration vector.

By solving Equation (6), the position of the clump center of mass at moment $t$ can be expressed by as follows:

$$
x_{i}^{(t+\Delta t)}=x_{i}^{(t)}+\dot{x}_{i}^{(t+\Delta t / 2)} \Delta t .
$$

After determining the translational and rotational velocities of the clump, the velocity of each ball in the clump can be expressed as follows:

$$
\dot{x}_{i}^{[\mathrm{p}]}=\dot{x}_{i}^{[\mathrm{G}]}+\varepsilon_{i j k} \omega_{j}\left(x_{k}^{[\mathrm{p}]}-x_{k}^{[\mathrm{G}]}\right) .
$$

The above iterative calculations were continuously performed on all elements so that the dynamic simulation of the studied object can be finally realized.

2.3. Numerical Verification. The correctness and availability of the numerical simulations need to be verified before performing systematic studies of the obstruction process of RNB toward granular flow. Numerical simulations were performed on the basis of the experiments conducted by Jiang and Towhata [45]. These experiments were mainly carried out using the setup shown in Figure 2. A flow flume with a length, width, and height of $2.93 \mathrm{~m}, 0.30 \mathrm{~m}$, and $0.35 \mathrm{~m}$, respectively, was used to provide an experimental environment for dry granular flow movement, and the angle between the bottom of the flow flume and the horizontal plane was adjusted by rotation around the bottom fixed 


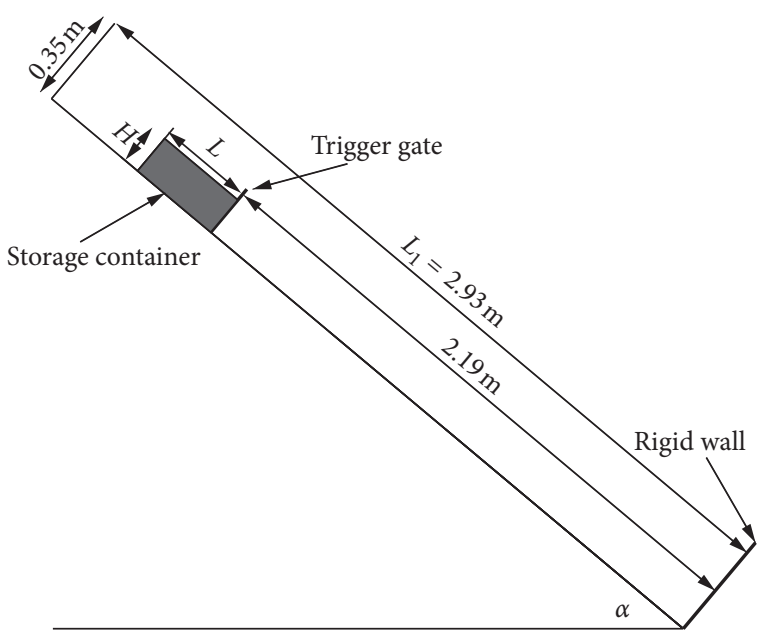

Figure 2: Sketch of the experimental equipment.

point. At the downstream end of the flow flume, a rigid wall was placed perpendicular to the bottom. The normal force on the wall could be measured by the sensor. At the initial moment, the dry granular material was placed in a square box of the upper flow flume. The dry granular material was limestone gravel with a grain size of $20 \mathrm{~mm}$. $L$ and $H$ are the length and height of the box, respectively. During the experiment, the start of the granular material was controlled by momentarily opening the trigger gate perpendicular to the flow flume, and the distance $L_{1}$ between the control gate and the rigid wall was $2.19 \mathrm{~m}$. The friction angles of the flume bottom, flume sides, and rigid wall were $25^{\circ}, 15^{\circ}$, and $21^{\circ}$, respectively, which were obtained from experiments [45]. Based on these friction angles, the corresponding friction coefficients were determined.

The downstream rigid wall was divided into 6 segments, with each having the same width of $0.3 \mathrm{~m}$ as the bottom board of the flow flume. The height of segment nos. 1-5 was $5 \mathrm{~cm}$, and the height of segment no. 6 was $15 \mathrm{~cm}$, as shown in Figure 3. Although granular flow can run up after reaching the rigid wall, the grains that can reach the height of segment 6 under the experimental conditions may be quite few, which will lead to a quite limited impact of the grains at this height. Therefore, the height of segment 6 being set to 3 times that of the other segments can facilitate the collection of the impact force of these scattered grains so that the sensor can effectively work within its measuring range. The unit-width normal force of the six segments from the bottom to the top is denoted by $F_{i}$, where $i$ is the number of each horizontal segment, and segments were numbered from the bottom to the top in the order of $1-6 . h_{i}$ represents the distance between the center of each horizontal segment and the bottom.

As the actual grain shape is generally irregular, the simulation of the movement process of granular flow using only spherical grain element sometimes does not achieve good results $[24,30]$. To provide a more realistic effect, an irregular shape of limestone gravel comprising four balls of the same size was used in the experiments. The four balls formed a clump element, and the distance between any two

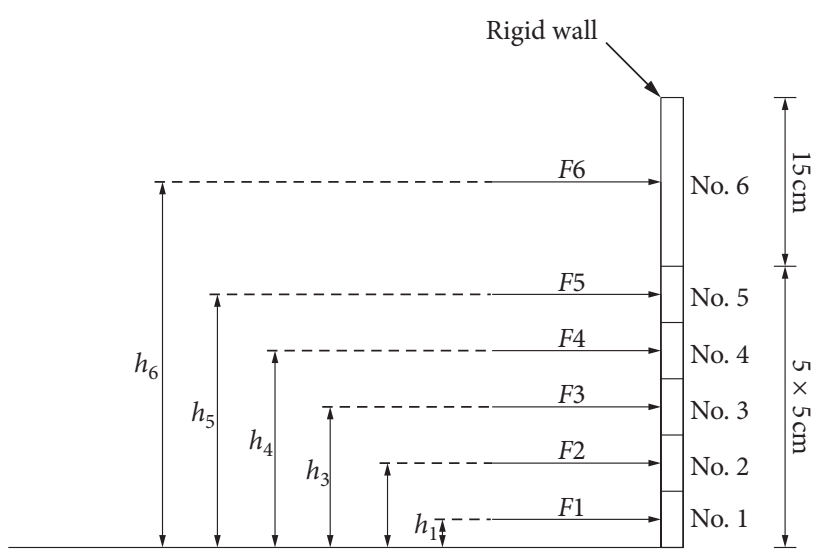

FIgURE 3: Sketch of the force acting on the rigid wall.

of the four balls was equal. After using the test data for numerical calibration and optimization, it was determined that when the distance $D$ between the centers of the balls is 1.5 times the radius $R$ of the balls (i.e., $D=1.5 R$ ), the simulation effect is the best [30], as shown in Figure 4. To make the clump element and the limestone gravel with a diameter of approximately $20 \mathrm{~mm}$ equivalent in volume and density, the radii and densities of the balls were calibrated. It should be specially noted that the equivalent volume method was chosen here instead of the equivalent size method because when the clump mass is the same as the grain mass, the equivalent volume method enables the clump to achieve a density consistent with limestone gravel for a realistic and efficient numerical simulation. The radius of the balls was finally determined to be $6.6 \mathrm{~mm}$, and the density was $2.34 \mathrm{~kg} / \mathrm{m}^{3}$.

According to the physical property parameters of reference [45], the mesoparameters shown in Table 1 were used in the calculation.

To make the number of clump elements in the simulation approximately equal to the number of gravel grains in the experiment, the number of clumps np was calculated using equation $n_{p}=V_{t} \rho_{t} / V_{s} \rho_{s}$, where $V_{t}$ and $\rho_{t}$ are, respectively, the total volume and density of the limestone gravel and $V_{s}$ and $\rho_{s}$ are the volume and density of the clumps, respectively.

The experiment numbered $L 44-H 15-\alpha 40^{\circ}$ in the literature [45] was selected for numerical calculation and verification. This experiment requires $L=44 \mathrm{~cm}, H=15 \mathrm{~cm}$, and $\alpha=40^{\circ}$ in Figure 2 .

Before the numerical verification, the mesoscopic parameters were calibrated by means of calculation and adjustment to correspond to the macroparameters used in the literature [45], such as the density and contact friction angle. In addition, to establish the calculation model, the size of the flow flume and the initial position and shape of the granular material (including the length and height) were calibrated before the numerical verification to ensure that the calculation corresponded to the experimental conditions of the geometric model. After the calibration work was done, the variation of the normal forces acting on the six rigid walls in the experiment was calculated using the discrete element 


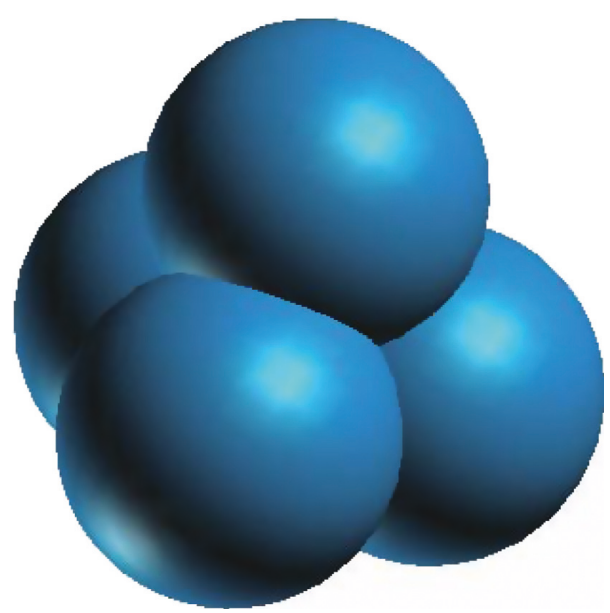

Figure 4: Clump discrete element.

TABLE 1: Mesoparameters used in the simulation.

\begin{tabular}{|c|c|c|c|c|c|c|c|c|}
\hline $\begin{array}{l}\text { Clump } \\
\text { normal } \\
\text { stiffness kn } \\
\left(\mathrm{N} \cdot \mathrm{m}^{-1}\right)\end{array}$ & $\begin{array}{l}\text { Clump shear } \\
\text { stiffness ks } \\
\left(\mathrm{N} \cdot \mathrm{m}^{-1}\right)\end{array}$ & $\begin{array}{l}\text { Density of the balls } \\
\text { forming a clump } \\
\qquad\left(\mathrm{kg} \cdot \mathrm{m}^{-3}\right)\end{array}$ & $\begin{array}{l}\text { Clump } \\
\text { friction } \\
\text { coefficient }\end{array}$ & $\begin{array}{c}\text { Wall normal } \\
\text { stiffness kn } \\
\left(\mathrm{N} \cdot \mathrm{m}^{-1}\right)\end{array}$ & $\begin{array}{l}\text { Wall shear } \\
\text { stiffness ks } \\
\left(\mathrm{N} \cdot \mathrm{m}^{-1}\right)\end{array}$ & $\begin{array}{l}\text { Bottom } \\
\text { friction } \\
\text { coefficient }\end{array}$ & $\begin{array}{l}\text { Friction } \\
\text { coefficient of } \\
\text { the sidewall }\end{array}$ & $\begin{array}{l}\text { Friction } \\
\text { coefficient of } \\
\text { the rigid wall }\end{array}$ \\
\hline $1 e 4$ & $1 e 4$ & $2.34 e 3$ & 0.47 & $1 e 8$ & $1 e 8$ & 0.47 & 0.27 & 0.38 \\
\hline
\end{tabular}

method, and the calculation results were compared with the experimental results, as shown in Figure 5. Since the raw data calculated based on the discrete element method are relatively fluctuating, noise reduction measures were taken to better compare and analyze the raw data and the experimental data.

In addition to the unit-width normal forces of each barrier, the total force and moment acting on the rigid wall could be further obtained, as shown in Figure 6, which includes the experimental [45] and simulated results of the total unit-width normal force $\mathrm{F}$ and moment $M$ of the test L44-H15- $\alpha 40^{\circ}$.

By comparing the numerical results with the experimental data, it can be seen that although there are certain differences in some details, the overall calculation results agree well with the experimental data. Numerical simulations can reflect the effect of granular flow on rigid walls [30]. Therefore, more in-depth research and discussion can be carried out on this basis.

\section{Numerical Results}

After verification of the numerical calculation, more information was also obtained, such as the force acting on the barrier from granular flow during the obstruction process. This also suggests that the numerical simulation can be used to study the obstruction of granular flow by some barriers that are difficult to examine using modern technology testing (such as RNB). In this study, the obstruction process of RNB toward granular flow was mainly analyzed. For the sake of comparison, the following numerical calculation studies were carried out with the calculation parameters and relevant conditions of the test $L 44-H 15-\alpha 40^{\circ}$, and the selected grains had the same size as in the verification experiment. At the same time, considering the direct and important influence of the masses of grains that have not been obstructed by the RNB in the downstream, the obstruction efficiency is defined in this study as the ratio of the mass of the grains obstructed by the RNB relative to the total mass of the grains sliding from the source area.

On the basis of the verification examples, the RNB was placed at different positions of the flow flume to obstruct the granular flow to allow study of the force characteristics and the obstruction efficiency of the RNB under different setting conditions, as shown in Figure 7. For easy comparative analysis, the calculation parameters used for the numerical verification were still adopted in the following study. In particular, the RNB was simulated by line-wall elements. The linear contact model and slip model were used for the line wall elements as well as the wall elements, and the material property setting of the line wall elements was the same as that of the sidewall (Table 1).

The RNB shown in Figure 7 has a width of $0.3 \mathrm{~m}$, which is the same as the width of the flow flume. In the following experiments, the obstruction effect of the RNB under different conditions (such as different positions, heights, and mesh sizes) and the force characteristics of the RNB were studied and analyzed.

3.1. Influence of RNB Position. To study the influence of the $\mathrm{RNB}$ position on the dynamic process, the RNB was placed $0.55 \mathrm{~m}, 1.10 \mathrm{~m}$, and $1.64 \mathrm{~m}$ away from the downstream rigid wall, respectively. At the same time, to obtain good 


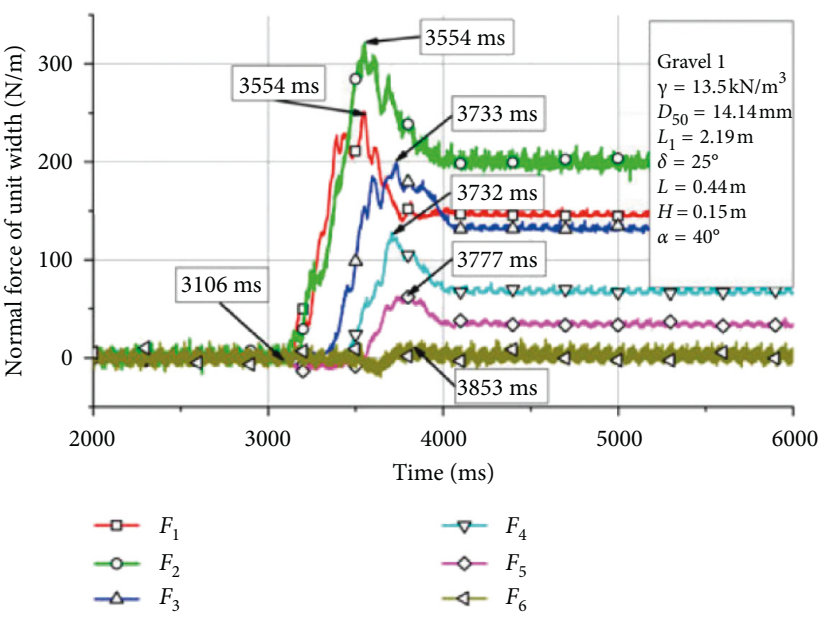

(a)

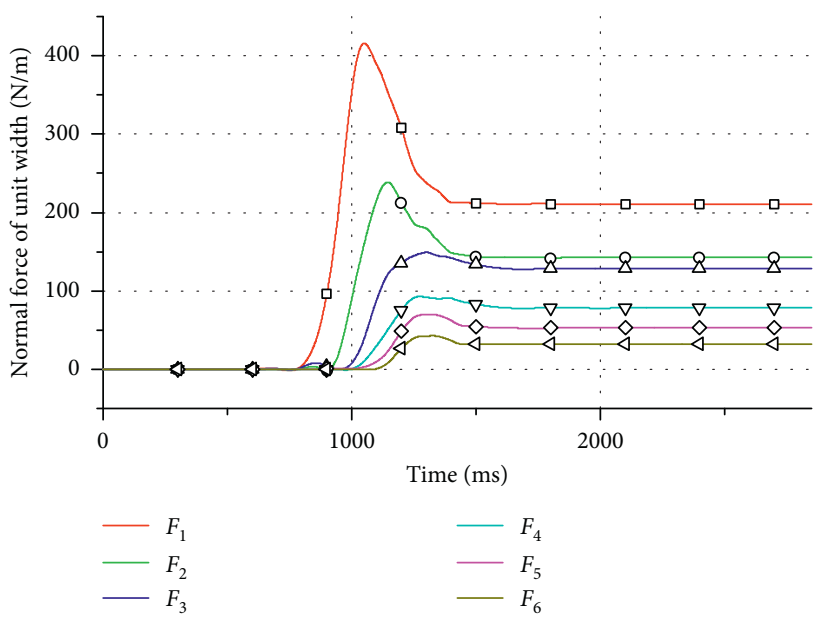

(b)

FIGURE 5: Time history of the unit-width normal force $\left(L 44-H 15-\alpha 40^{\circ}\right)$ : (a) the experimental data [45]; (b) the numerical results [30].

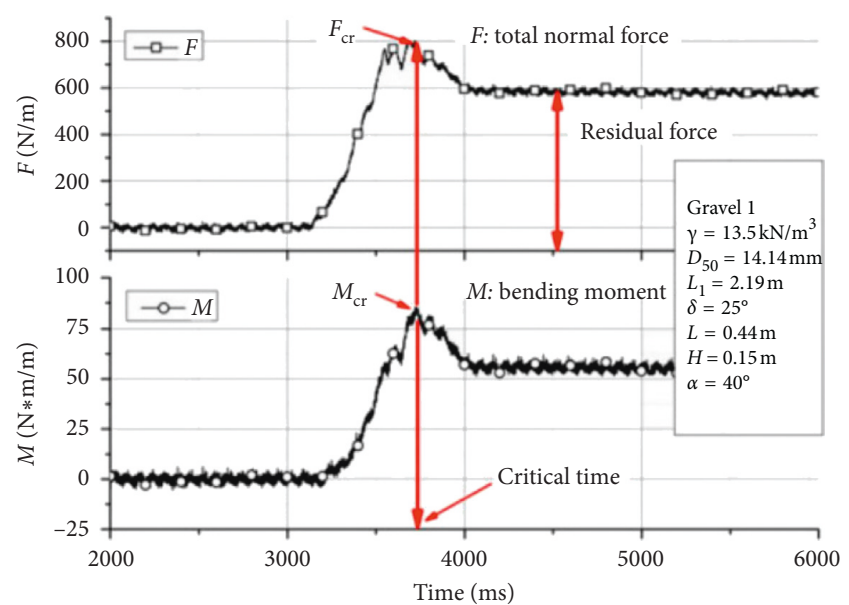

(a)

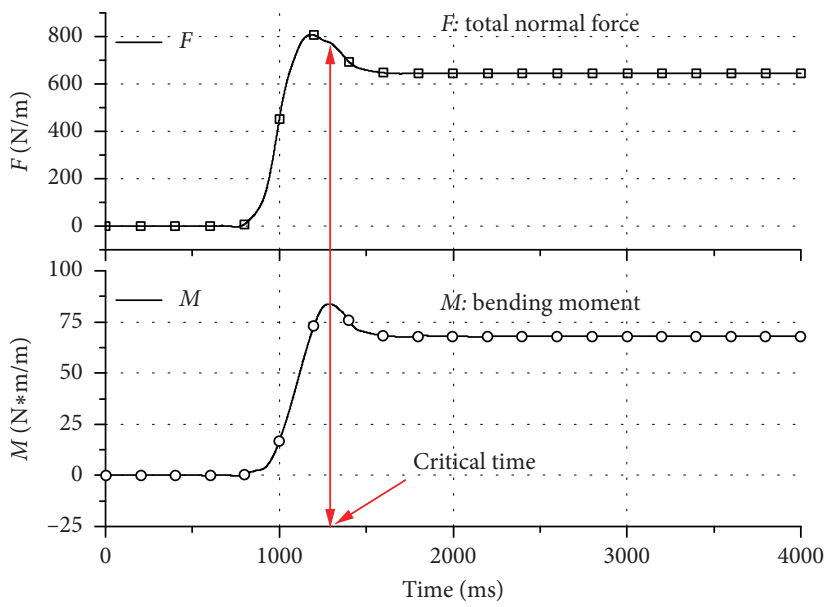

(b)

FIgURE 6: Time history of the total unit-width normal force and moment (L44-H15- $\alpha 40^{\circ}$ ): (a) the experimental data [45]; (b) the numerical results $[30]$.

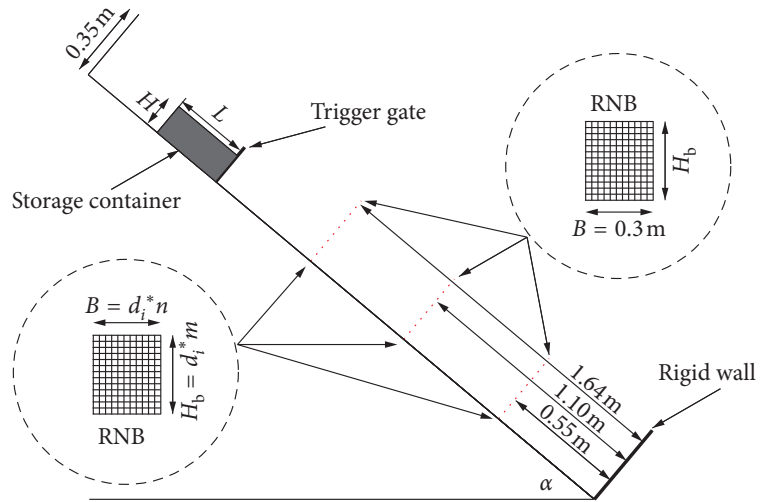

FIGURE 7: Setting of RNB in the flow flume.

obstruction efficiency, the RNB dimensions were set to $0.30 \mathrm{~m} \times 0.35 \mathrm{~m}$, which was consistent with the crosssectional dimension of the flow flume, and the mesh size was $20 \mathrm{~mm}$. During the numerical verification of the test for L44-H15-40 ${ }^{\circ}, 2408$ clumps matched with the test granular material were generated in the source area. These clumps were used in the following analysis of the uniform-size grains. The obstruction efficiency of the RNB toward the granular flow was calculated at different positions, as shown in Table 2.

It can be seen from Table 2 that, owing to the reasonable height and mesh size, the obstruction efficiency was over $98 \%$, which could basically achieve effective obstruction of the granular flow. The grains which were not effectively obstructed may have passed over the RNB by colliding with each other during the movement. When the RNB was placed at the relatively downstream position (in Case A1), the velocity of the grains was larger and the collision between the grains was more frequent, so the obstruction was less efficient than that when the RNB was placed upstream. Since each grain has its own velocity, to describe the overall 
TABLe 2: Obstruction efficiency of RNB at different positions.

\begin{tabular}{lcccc}
\hline & Distance from the rigid wall $(\mathrm{m})$ & Mesh size $(\mathrm{m})$ & Height $(\mathrm{m})$ & Obstruction efficiency $(\%)$ \\
\hline Case A1 & 0.55 & 0.02 & 0.35 & 98.01 \\
Case A2 & 1.10 & 0.02 & 0.35 & 98.48 \\
Case A3 & 1.64 & 0.02 & 0.35 & 98.88 \\
\hline
\end{tabular}

movement of the granular flow, the velocities of all grains were weighted and averaged, and then the granular flow velocity was obtained, which can be expressed as follows:

$$
v_{f}=\frac{\sum_{c=1}^{N_{c}} m_{i} v_{i}}{\sum_{c=1}^{N_{c}} m_{i}}
$$

where $v_{f}$ is the granular flow velocity and $m_{i}$ and $v_{i}$ are the mass and velocity of the $i^{\text {th }}$ clump, respectively.

The time history of the granular flow velocity when the RNB was placed at different positions was calculated, as shown in Figure 8.

The time history of the impact of the granular flow on the RNB when placed at different positions was calculated, as shown in Figure 9.

From the time history of the velocity when the RNB was placed at different positions, it can be seen that when the grains moved toward the RNB, the velocity decreased significantly due to the obstruction from the RNB, and the maximum velocity appeared accordingly. The maximum velocity is an important indicator to characterize the hazard of granular flow and is one of the significant control factors in engineering design. Compared with the case in which the RNB was placed upstream, the granular flow had a higher velocity (the maximum velocity) when it reached a downstream RNB, and its deceleration process was faster, which may cause a stronger impact on the downstream RNB. This deduction was also confirmed in Figure 9, where it can be clearly seen that when the RNB was placed upstream, the peak and stable values of the unit-width normal force and moment acting on the RNB were smaller, and the ratios between the two values were also smaller. When the RNB was placed downstream, the impact of the granular flow was stronger due to the greater velocity (the maximum velocity), so the peak and stable values of the unit-width normal force and moment acting on the RNB, as well as the ratio of the two values, were larger, and at this time, the impact on the RNB was more fluctuating.

To analyze the typical process of a granular flow under obstruction by an RNB and to understand the characteristics of the forces acting on the RNB more deeply, the Case A1 working condition with a large impact on the RNB was studied. The velocity and shape of the granular flow at different moments are shown in Figure 10.

As seen from Figure 10, when the grains reached the $\mathrm{RNB}$, a reverse rebound motion occurred. With the arrival and accumulation of the grains, a "dead zone" with a granular velocity of zero was formed in front of the RNB. This region actually reshaped the bottom boundary of the granular flow movement, and the following grains began to "run-up." As the dead zone progressively expanded and the grains continued to run up on the dead zone, the final

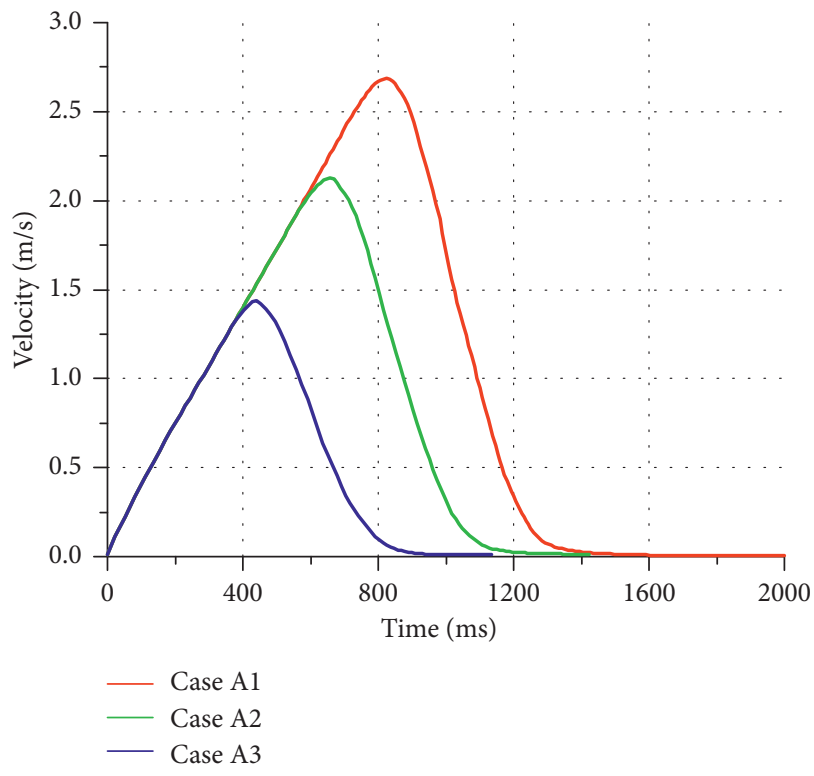

FIgURE 8: Time history of the granular flow velocity when the RNB was placed at different positions.

accumulation formed in front of the RNB. It has also been found that several grains passed over the RNB due to an upward velocity generated by the strong collision and then impacted the downstream rigid wall. The downstream RNB obstructed the grains that had larger velocities and stronger collisions, and these grains also had higher run-up heights and final stack heights, with more significant run-up motion. This is a good explanation for the fact that the peak and stable values of the unit-width normal force and moment acting on the RNB were larger and that the time history was more fluctuating.

In actual protection engineering, it is often impossible to determine the source of the failure rock and soil material. For example, the location of an unstable landslide on the side of a gully is difficult to determine effectively. Moreover, an RNB in a higher position has a large potential energy after obstructing a granular flow multiple times, and once destroyed, it will pose a greater threat downstream. Therefore, to obtain a greater range of protection, the RNB is generally placed as close to the downstream as possible to more reliably protect the buildings and facilities. Therefore, the following calculations were based on the setting of a downstream RNB.

3.2. Influence of RNB Height. To study the influence of the height setting of the RNB on the dynamic process, RNB with heights of $0.10 \mathrm{~m}, 0.20 \mathrm{~m}, 0.30 \mathrm{~m}$, and $0.35 \mathrm{~m}$ was placed $0.55 \mathrm{~m}$ away from the rigid wall, respectively. The width of 


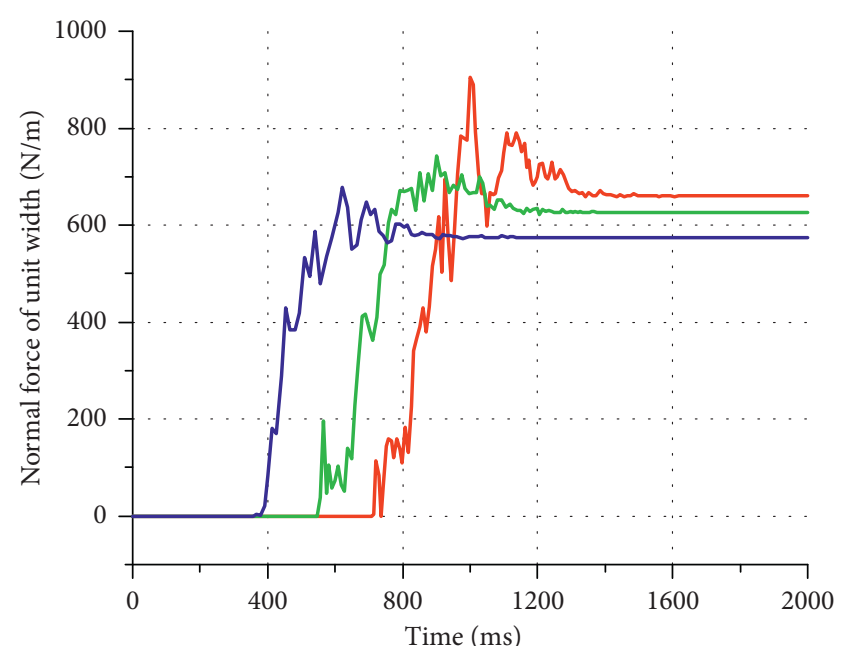

- Case A1

- Case A2

- Case A3

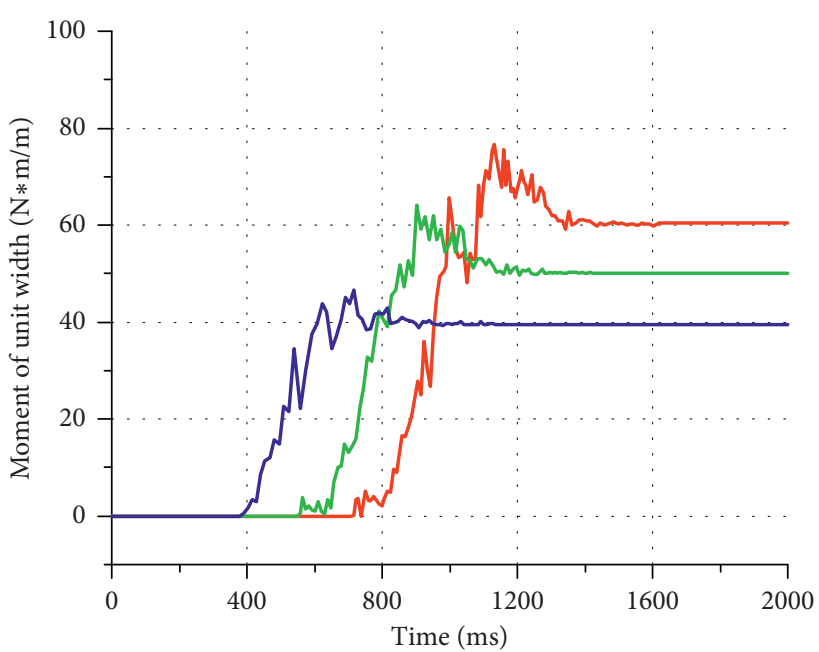

- Case A1

- Case A2

- Case A3

(a)

(b)

FIGURE 9: Time history of the impact of the granular flow on the RNB when placed at different positions: (a) time history of the unit-width normal force; (b) time history of the unit-width moment.

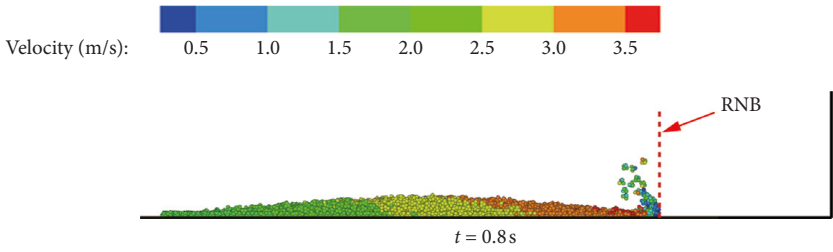

(a)

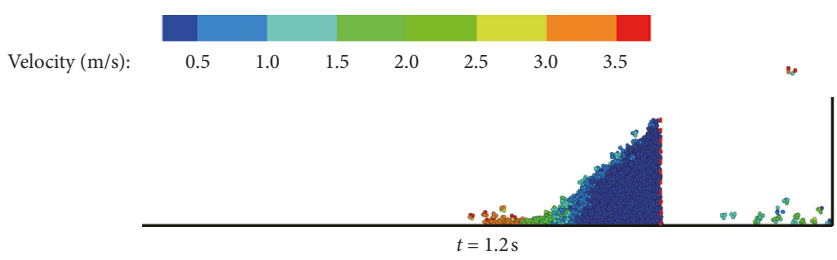

(c)

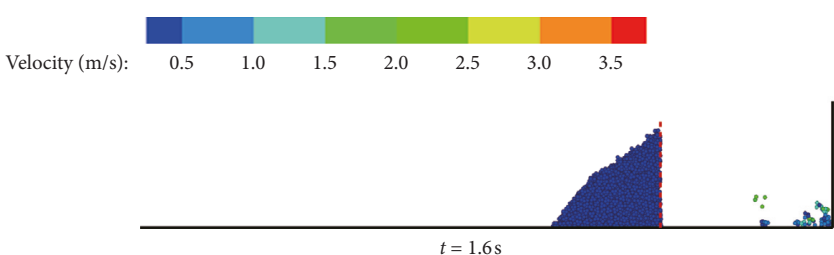

(e)

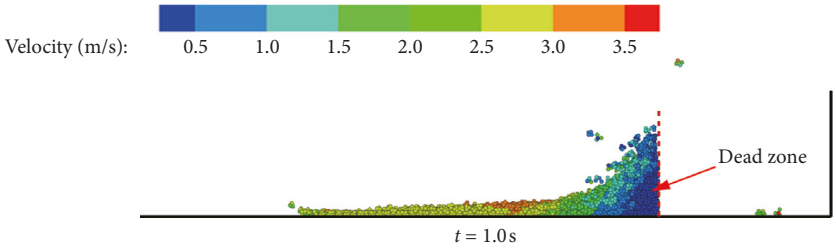

(b)

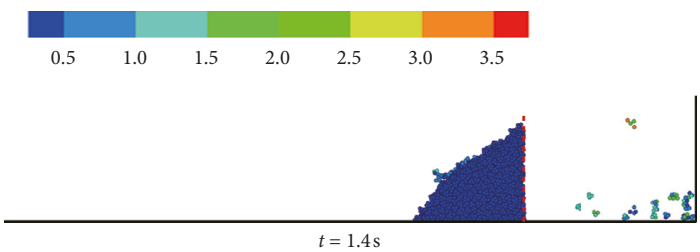

(d)

$\begin{array}{llllllll}\text { Velocity }(\mathrm{m} / \mathrm{s}): & 0.5 & 1.0 & 1.5 & 2.0 & 2.5 & 3.0 & 3.5\end{array}$

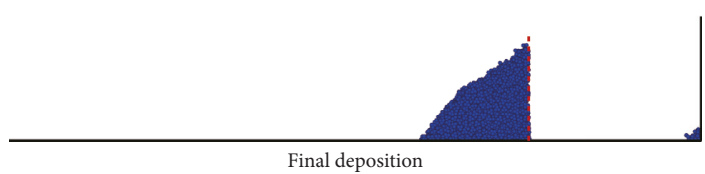

(f)

Figure 10: Granular flow process in Case A1.

the RNB was set to $0.30 \mathrm{~m}$, which was the same as the section width of the flow flume. The mesh size was also set to $20 \mathrm{~mm}$, and the obstruction efficiency of the RNB toward granular flow was calculated for different heights, as shown in Table 3.

It can be seen from Table 3 that the height of the RNB has a great influence on the obstruction efficiency. If a higher obstruction efficiency is desired, a sufficient RNB height is necessary, in addition to a reasonable mesh size. If the RNB is not high enough, a space sufficient to accommodate the granular material cannot be formed so that a high obstruction efficiency cannot be achieved. Meanwhile, since the grains run up after reaching the RNB, an "overflow" phenomenon may occur when the run-up height exceeds the RNB height, resulting in a low obstruction efficiency. 
TABLE 3: Obstruction efficiency of RNB with different heights.

\begin{tabular}{lcccc}
\hline & Distance from the rigid wall $(\mathrm{m})$ & Mesh size $(\mathrm{m})$ & Height $(\mathrm{m})$ & Obstruction efficiency $(\%)$ \\
\hline Case B1 & 0.55 & 0.02 & 0.10 & 37.04 \\
Case B2 & 0.55 & 0.02 & 0.20 & 83.86 \\
Case B3 & 0.55 & 0.02 & 0.30 & 97.82 \\
Case B4 & 0.55 & 0.02 & 0.35 & 98.01 \\
\hline
\end{tabular}

The time history of the granular flow velocity when RNB with different heights was placed was calculated, as shown in Figure 11.

The time history of the impact of the granular flow on the placed RNB with different heights was calculated, as shown in Figure 12.

It can be seen from Figure 11 that, under the obstruction of the RNB, the velocity began to decrease. Each case has the same maximum granular flow velocity, and the granular flow has the same deceleration process before running up to the RNB height. Interestingly, however, when the RNB height was insufficient, an obvious "platform" appeared in the falling section of the velocity curve, which means that the decrease of the velocity slowed, which was related to the overflow. During the run-up, once the grains reached the maximum height of the RNB, they passed over the RNB and continued to accelerate downstream. If the RNB height exceeded the maximum run-up height of the granular flow, overflow could be avoided. The increase of the RNB height above this maximum run-up height had little effect on the dynamic process. In this case, increasing the RNB height can only obstruct a very small number of grains splashed by the collision, and the influence on the obstruction efficiency was extremely small.

It can be seen from Figure 12 that when the RNB height exceeded the maximum run-up height of the granular flow, the time history of the impact on the RNB did not substantially change. When the RNB height was relatively small, although the granular flow had the same maximum velocity as in the cases in which the RNB was higher, a large number of grains passed over the RNB in an overflow manner, thereby reducing the impact on the RNB. During the overflow, the ratios of the peak and stable values of the unitwidth normal force and moment acting on the RNB were relatively large. By comparing the time histories of Case B1 and Case B2, when overflow occurs, the peak and stable values of the unit-width normal force and moment acting on the RNB are not simply proportional to the RNB height. At the same time, as the RNB height increases, the run-up height of the grains is larger, and the force arm increases so that the growing rate of the unit-width moment acting on the RNB is greater than that of the unit-width normal force.

To analyze the kinetic characteristics of a granular flow with an insufficient RNB height, the Case B2 working condition with typical overflow characteristics was studied in detail, and the velocity and shape of the granular flow at different moments are shown in Figure 13.

As seen from Figure 13, the rebound of the grains when they first reached the RNB and the formation of the dead zone in front of the RNB showed no difference relative to the discussed Case A1. After the subsequent grains ran up to the

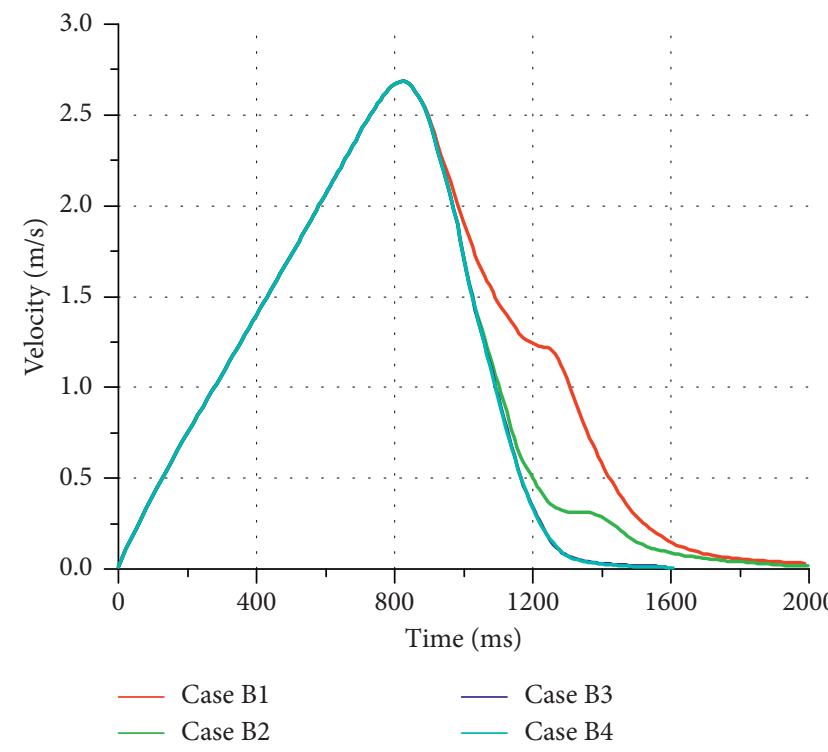

Figure 11: Time history of the granular flow velocity when RNB with different heights was placed.

maximum height of the RNB, they passed over the RNB in an overflow manner due to the lack of sufficient constraints. A stable dead zone was also gradually formed during the overflow process, and the dead zone was continuously developed and formed a final accumulation. After the overflow, the discharged grains moved downstream in the form of a projectile, which caused a large impact on the downstream bottom area of the flow flume. In actual engineering, this area may eventually form an impact crater, so it needs to be an item of focus. The calculation results show that, under the condition of an insufficient RNB height, the grains pass the $\mathrm{RNB}$ in the manner of overflow, thereby causing damage to the downstream. Due to the complexity of the granular flow movement and application conditions, the design height of the RNB cannot be obtained by simple calculation. The most effective method is to obtain the maximum run-up height of the granular flow under the corresponding conditions by numerical calculation.

According to the above analysis, the granular flow runs up when obstructed by the RNB, and overflow occurs when the RNB height cannot reach the maximum run-up height. Therefore, only if the design height of the RNB exceeds the maximum run-up height of the granular flows, the overflow can be avoided, and then the RNB can play its due role. Under the conditions of the examples in this study, to achieve better obstruction efficiency, the RNB height should be at least $0.3 \mathrm{~m}$. Combined with the size of the actual flow flume, the following calculations and discussion will all be based on an RNB height of $0.35 \mathrm{~m}$. 


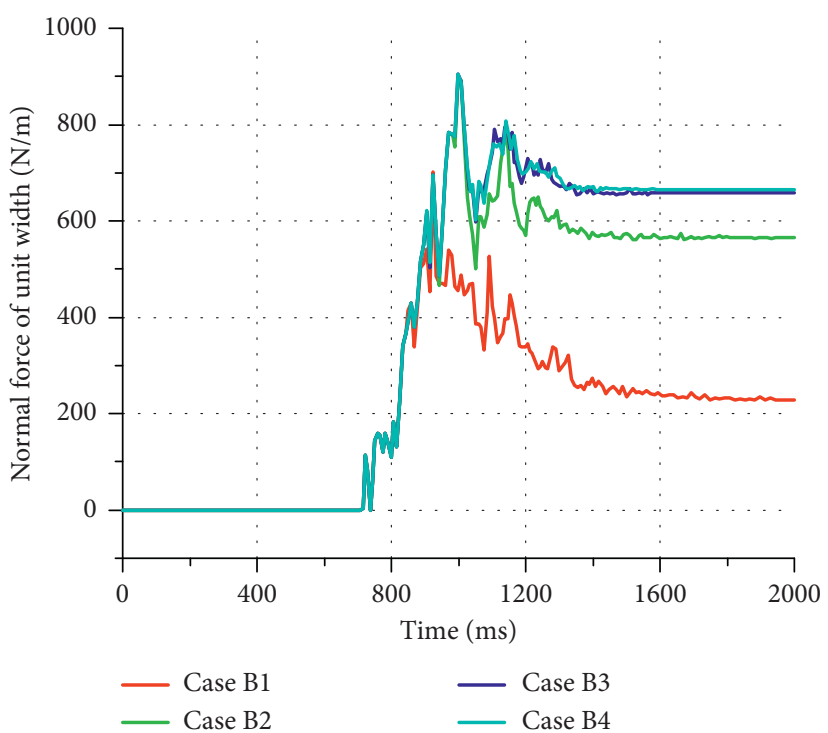

(a)

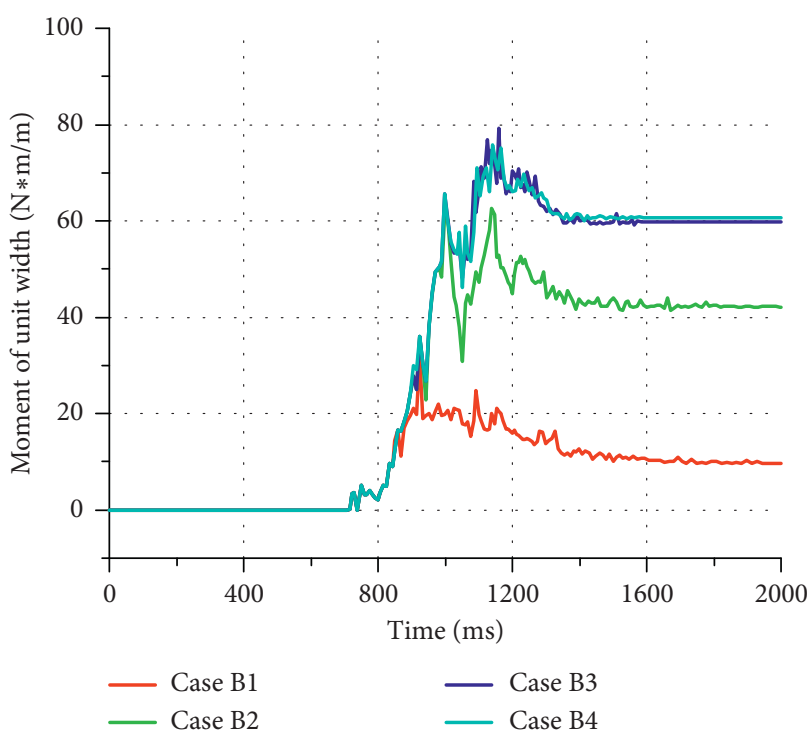

(b)

FIGURE 12: Time history of the impact of the granular flow on RNB with different heights: (a) time history of the unit-width normal force; (b) time history of the unit-width moment.

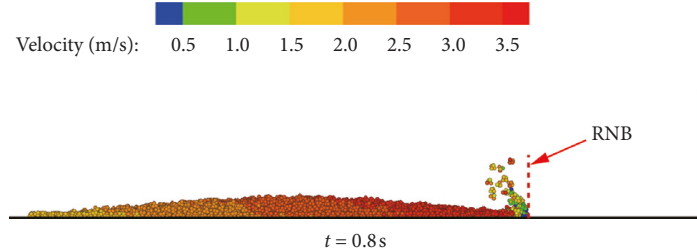

(a)

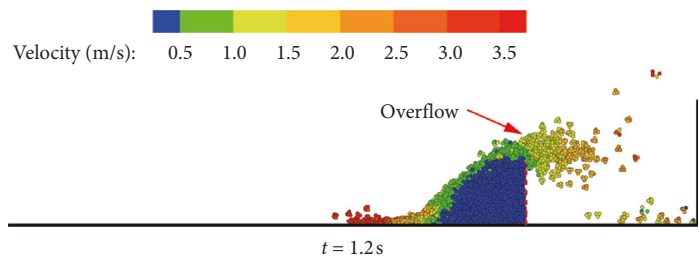

(c)

Velocity $(\mathrm{m} / \mathrm{s}): \quad 0.5 \quad 1.0 \quad 1.5 \quad 2.0 \quad 2.5 \quad 3.0 \quad 3.5$

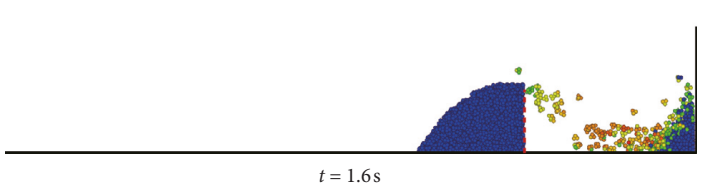

(e)

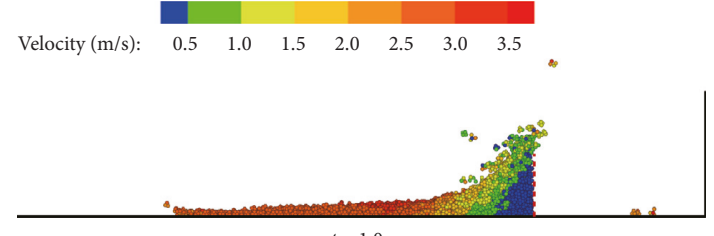

$t=1.0 \mathrm{~s}$

(b)
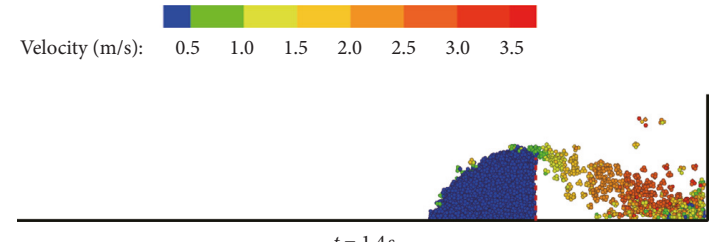

(d)
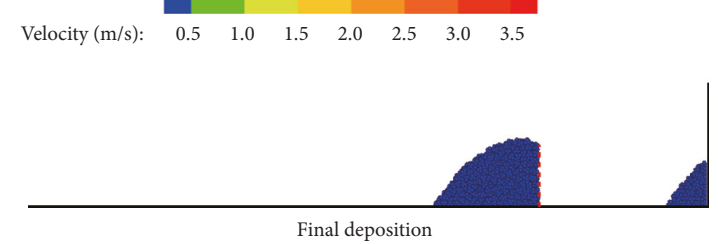

(f)

Figure 13: Granular flow process in Case B2.

3.3. Influence of the RNB Mesh Size. To study the influence of the RNB mesh size on the dynamic process, an RNB with a size of $0.30 \mathrm{~m} \times 0.35 \mathrm{~m}$ was placed $0.55 \mathrm{~m}$ away from the rigid wall. The mesh size was set to $10 \mathrm{~mm}, 20 \mathrm{~mm}, 30 \mathrm{~mm}$, and $40 \mathrm{~mm}$, respectively. The obstruction efficiency of the RNB toward granular flow was calculated for different mesh sizes, as shown in Table 4.
Table 4 shows a high obstruction efficiency of the RNB for the set mesh sizes. Even when the mesh size reached nearly 2 times the grain size, the obstruction efficiency was still relatively high, reaching $78.70 \%$, which seems to be different from our intuitive experience. For deeper understanding of this phenomenon, a further analysis is necessary. In the case in which the mesh size is larger than 
TABle 4: Obstruction efficiency of the RNB with different mesh sizes.

\begin{tabular}{lcccc}
\hline & Distance from the rigid wall $(\mathrm{m})$ & Mesh size $(\mathrm{m})$ & Height $(\mathrm{m})$ & Obstruction efficiency $(\%)$ \\
\hline Case C1 & 1.10 & 0.01 & 0.35 & 99.90 \\
Case C2 & 1.10 & 0.02 & 0.35 & 98.00 \\
Case C3 & 1.10 & 0.03 & 0.35 & 90.20 \\
Case C4 & 1.10 & 0.04 & 0.35 & 78.70 \\
\hline
\end{tabular}

the grain size, although the grains that first reach the RNB may pass through the RNB due to their smaller size, the subsequently arriving grains form a force chain by contacting each other in front of the RNB. The force chain can effectively prevent the continuous passage of the grains, thereby achieving effective obstruction of grains smaller than the mesh size and forming the mechanism through which an RNB with a large-mesh size can still effectively obstruct fine grains. When the mesh size is relatively small, unobstructed grains mainly pass over the RNB by collision, which is sometimes difficult to avoid in actual engineering. When the mesh size reaches $0.02 \mathrm{~m}$, continuing to reduce the mesh size provides stronger constraints and hindrance to the grains and, thus, can slightly improve the obstruction efficiency.

The time history of the granular flow velocity when RNB with different mesh sizes was placed was calculated, as shown in Figure 14.

The time history of the impact of the granular flow on RNB with different mesh sizes was calculated, as shown in Figure 15.

It can be seen from Figure 14 that the granular flow has different velocity curves after reaching RNB with different mesh sizes. When the mesh size is relatively large, the velocity deceleration is slow, and even the peak of the velocity curve is slightly increased because more grains passed through the RNB with a larger mesh size to continue the acceleration downstream. Obviously, the maximum granular flow velocity before reaching the RNB should be adopted for structural design. This value is the same in these cases because the increase in velocity caused by the accelerated motion of the grains that passed through the mesh to the downstream is not related to the force acting on the RNB. When the mesh size reached the grain size, the influence of reducing the mesh size on the velocity was not obvious.

It can be seen from Figure 15 that as the mesh size increased, part of the grains passed through the larger mesh, so the unit-width normal force and moment acting on the RNB were greatly reduced. Although this phenomenon reduced the load on the RNB, the grains passing through the mesh may also harm the downstream. When the mesh size reached $0.02 \mathrm{~m}$, which was equivalent to the grain size, continuing to reduce the mesh size hardly changed the time history of the impact on the RNB, and this was because when the mesh size was small enough, the grains could no longer pass through the RNB. When the mesh size was small enough, the granular flow had a higher run-up and, thus, a larger force arm, resulting in a larger difference between the unit-width moments acting on the RNB with different mesh sizes.

To analyze the kinetic characteristics of the granular flow under the condition of a larger mesh size RNB, Case C3 with grain passing-through characteristics was studied in detail,

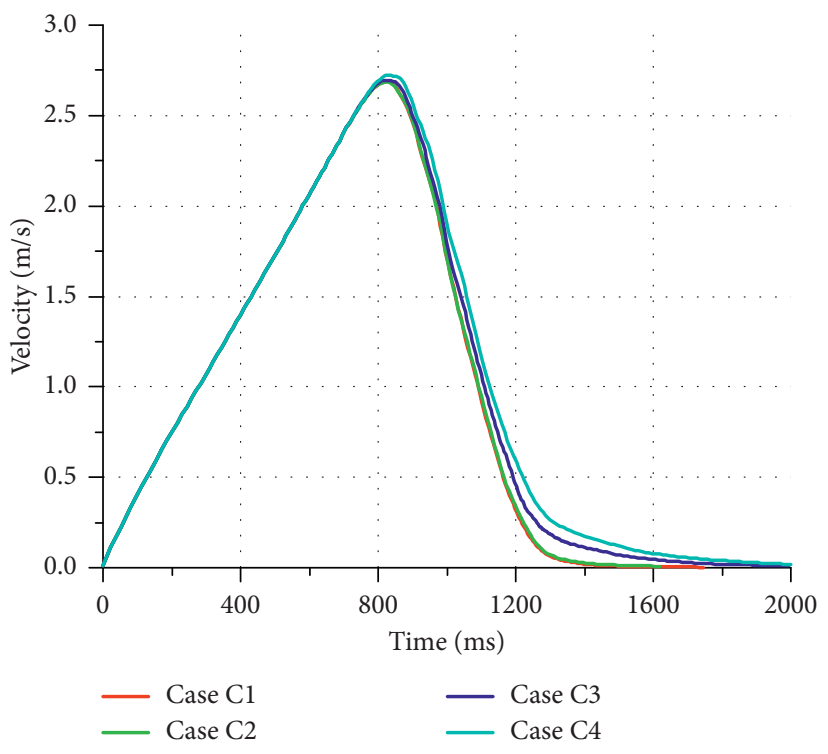

FIGURE 14: Time history of the granular flow velocity when RNB with different mesh sizes was placed.

and the velocity and shape of the granular flow at different moments are shown in Figure 16.

It can be seen from Figure 16 that when the grains reached the RNB, since the grain size was smaller than the mesh size, part of the grains moved downstream through the RNB, and the grains that had not passed through the RNB contacted and supported each other to form a relatively stable force chain, which in turn formed a dead zone with an obstruction effect, after which the subsequent grains ran up in the dead zone. When the granular flow reached a new height, some of the grains moved downstream through the RNB, so the grains could be seen to pass through the RNB from the bottom to the top. The grains that passed through the RNB from a higher position moved downstream in the form of projectile motion. Similar to the overflow, the grains passing through the RNB from a higher position impacted the downstream bottom, and thus, the area of the impacted bottom should be given sufficient attention. Although the grain passing-through phenomenon exists when the mesh size is larger than the grain size, the force chain formed by the grains in front of the RNB can effectively obstruct the subsequent grains, thereby achieving better obstruction efficiency. However, due to the complexity of granular flow movement, it is impossible to obtain the obstruction efficiency under large-mesh size conditions by simple methods. Therefore, numerical simulations may be the most effective method when experimental conditions are limited. 


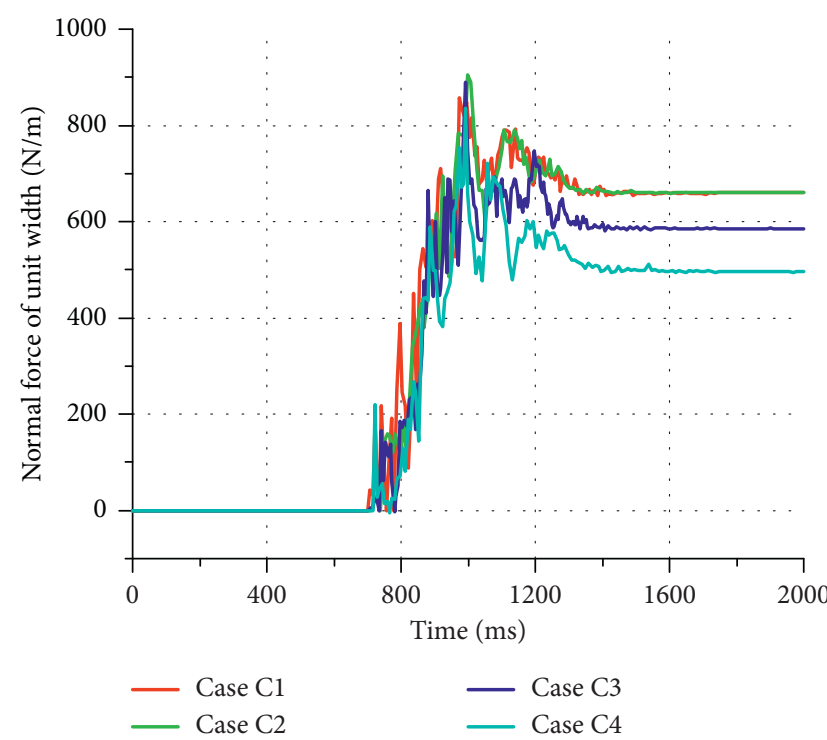

(a)

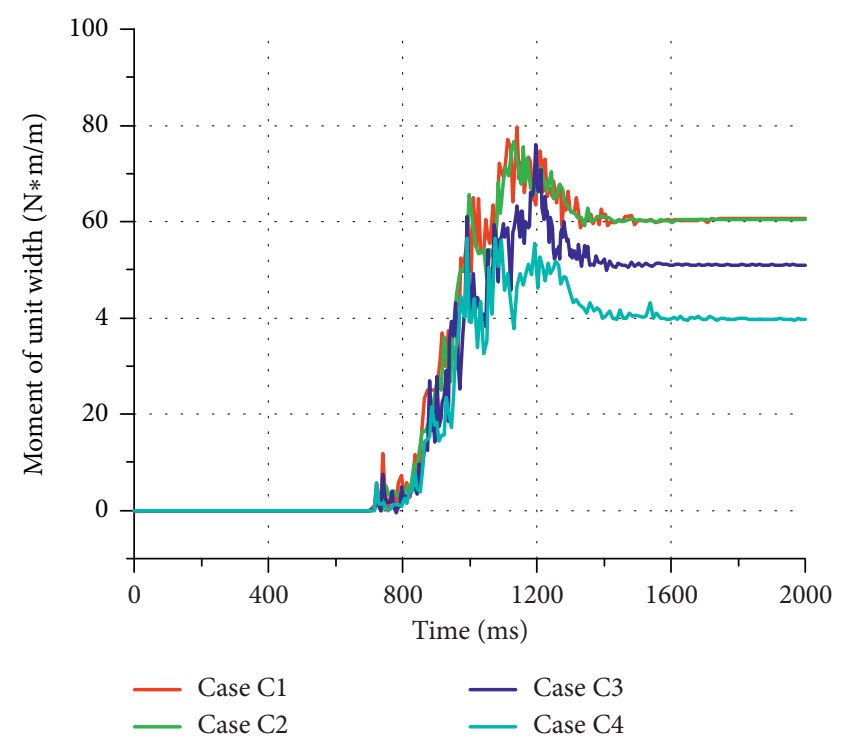

(b)

FIGURE 15: Time history of the impact of the granular flow on RNB with different mesh sizes: (a) time history of the unit-width normal force; (b) time history of the unit-width moment.

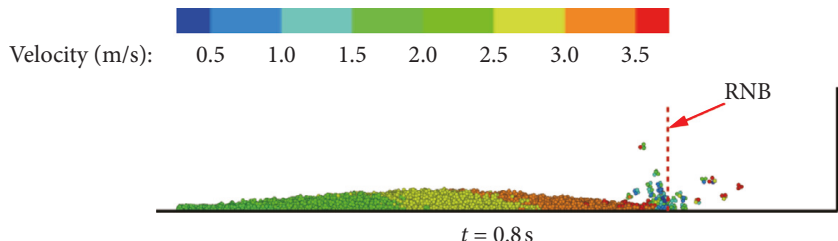

(a)

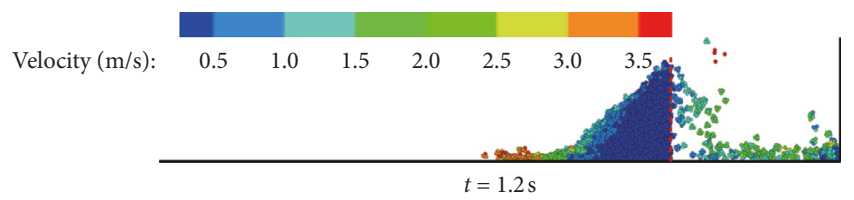

(c)

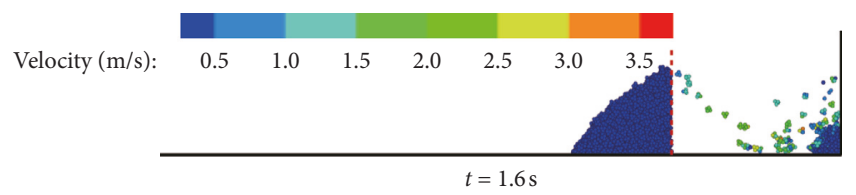

(e)

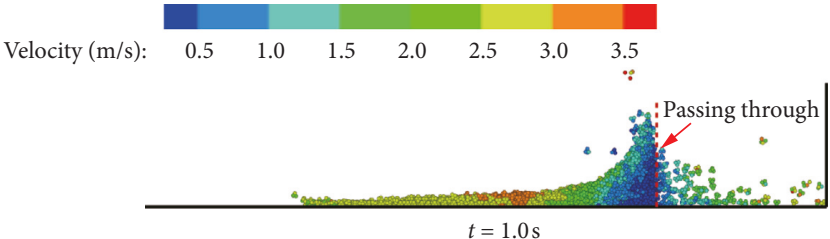

(b)

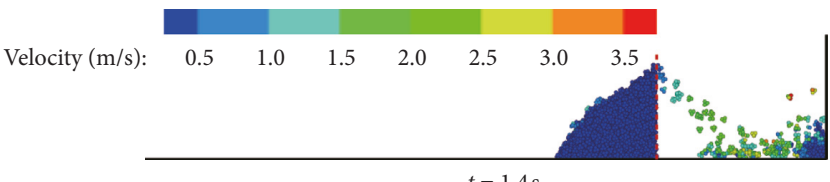

(d)

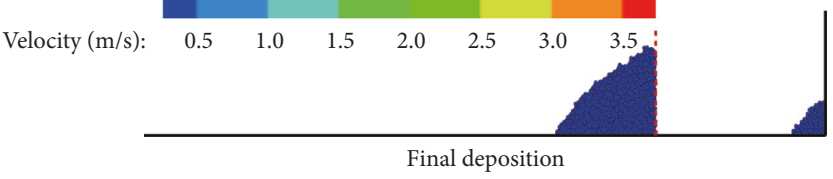

(f)

Figure 16: Granular flow process in Case C3.

The above analysis shows that, to improve the obstruction efficiency and avoid the grain passing-through phenomenon, it is necessary to set the mesh size small enough. In the case of uniform-size grains, if the mesh size used is smaller than the grain size, the passing-through phenomenon can be avoided, on the basis of which continuing to reduce the mesh size has little effect on improving the obstruction efficiency. However, grains in nature generally do not have the same size but instead have a wide gradation distribution, so further research is needed on the obstruction of the RNB toward graded grains.
3.4. Influence of Grain Size Gradation. To study the influence of the grain size gradation on the dynamic process, an RNB with a size of $0.30 \mathrm{~m} \times 0.35 \mathrm{~m}$ was placed $0.55 \mathrm{~m}$ away from the rigid wall to avoid the occurrence of overflow. The grains used in the calculations have a grain size gradation distribution, as shown in Table 5. Under the condition of test $L 44$ $H 15-\alpha 40^{\circ}$, a total of 13988 clumps were generated in the source area according to the grain size gradation distribution. These clumps used the same calculation parameters as those used for the uniform-sized grains. The mesh size of the RNB was set to $10 \mathrm{~mm}, 20 \mathrm{~mm}, 30 \mathrm{~mm}$, and $40 \mathrm{~mm}$, 
TABLE 5: Grain size gradation distribution.

\begin{tabular}{lcc}
\hline Grain size $(\mathrm{m})$ & Mass percentage & Cumulative mass percentage \\
\hline 0.010 & 5 & 5 \\
0.015 & 20 & 25 \\
0.020 & 50 & 75 \\
0.030 & 20 & 95 \\
0.040 & 5 & 100 \\
\hline
\end{tabular}

respectively. The obstruction efficiency of the RNB toward the granular flow was calculated under different mesh size conditions, as shown in Table 5.

Table 6 shows that although some grains were still able to pass through the RNB, RNB with larger mesh sizes can still obstruct smaller grains and obtain a higher obstruction efficiency. The formation mechanism of this result is similar to that by which RNB obstructs uniform-size grains. By comparing the results of Case $\mathrm{C}$ with those of Case $\mathrm{D}$, it can be found that, under the same mesh size conditions, when the mesh size was larger, the obstruction efficiency of the RNB toward the graded grains was higher, and when the mesh size was less, the obstruction efficiency was relatively low. This is because although the median size of the graded grains was the same as the grain size of the uniform-sized grains, when the mesh size was large, the RNB could ensure the obstruction of grains larger than the median size to improve the obstruction efficiency, but when the mesh size was small, the RNB could not effectively obstruct grains smaller than the median size, thereby reducing the obstruction efficiency. This indicates that the obstruction of an RNB toward the graded grains is quite different from that toward uniform-sized grains. Since the obstruction of graded grains mainly comes from the force chain formed by the mutual contact and support between the grains in front of the RNB, the obstruction efficiency of the RNB toward graded grains cannot be obtained by simple calculation.

The time history of the graded granular flow velocity when RNB with different mesh sizes was placed was calculated, as shown in Figure 17.

The time history of the impact of the graded granular flow on the placed RNB with different mesh sizes was calculated, as shown in Figure 18.

As seen from Figure 17, the time history of the graded granular flow velocity has similar characteristics to that of the uniform-sized grains. For example, when the mesh size was relatively large, the fine grains continued to move forward through the mesh and slowed the deceleration of velocity and caused a weak increase in the peak of the velocity curve, but the curve trends were not obvious. For this reason, although coarse grains exist as shown in Table 5, the larger mesh size was not discussed in this study. The fine grains that need to follow a more complex path to eventually pass through the RNB consume more energy, so the increased frequency collisions between the grading grains consume more energy too. Similar to the analysis of the cases with uniform-sized grains, although the peak of the velocity curve was obtained when studying the time history of the graded granular flow velocity under different RNB settings, it was affected by the accelerated motion of the grains that
TABLE 6: The obstruction efficiency of RNB with different mesh sizes toward graded grains.

\begin{tabular}{lcccc}
\hline & $\begin{array}{c}\text { Distance from the } \\
\text { rigid wall }(\mathrm{m})\end{array}$ & $\begin{array}{c}\text { Mesh } \\
\text { size }(\mathrm{m})\end{array}$ & $\begin{array}{c}\text { Height } \\
(\mathrm{m})\end{array}$ & $\begin{array}{c}\text { Obstruction } \\
\text { efficiency }(\%)\end{array}$ \\
\hline Case D1 & 0.55 & 0.01 & 0.35 & 99.73 \\
Case D2 & 0.55 & 0.02 & 0.35 & 97.32 \\
Case D3 & 0.55 & 0.03 & 0.35 & 89.26 \\
Case D4 & 0.55 & 0.04 & 0.35 & 82.02 \\
\hline
\end{tabular}

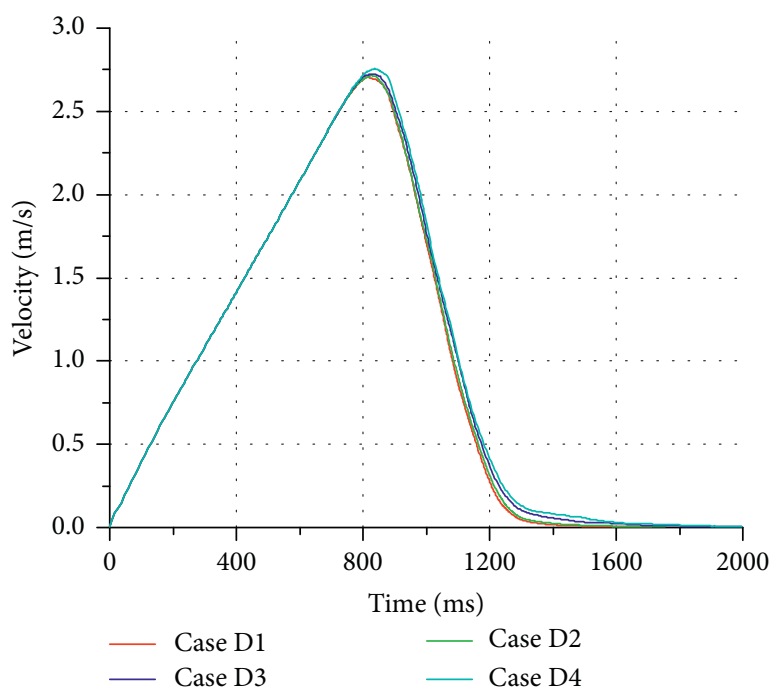

FIGURE 17: Time history of the graded granular flow velocity when RNB with different mesh sizes was placed.

passed through the RNB. Therefore, structural designs should be developed with reference to the maximum granular flow velocity before reaching the RNB but without consideration of the peak of the velocity curve, despite the quite small difference between these two velocity values in cases with graded grains.

It can be seen from the results shown in Figure 18 that the time histories of the impact of graded grains and uniform-sized grains on the RNB have similar characteristics. For example, the RNB with a larger mesh size under the impact of the graded grains was subject to a lesser unitwidth normal force and moment. The difference between the unit-width normal force and moment acting on the RNB is gradually reduced as the mesh size decreases. There are also some significant differences; for example, due to the greater density of the graded grains, the peak and stable values of the unit-width normal force and moment acting on the RNB were larger. Meanwhile, under the impact of graded grains, the unit-width normal force and moment acting on the RNB were more fluctuating. This strong fluctuation made it difficult to distinguish the local curves under different mesh size conditions. This is because under the impact of graded grains, the collisions between the grains were more complicated and frequent, so this strong fluctuation must also be paid attention in actual engineering.

During the flow process, the graded grains showed a regular grain size distribution through complex interactions 


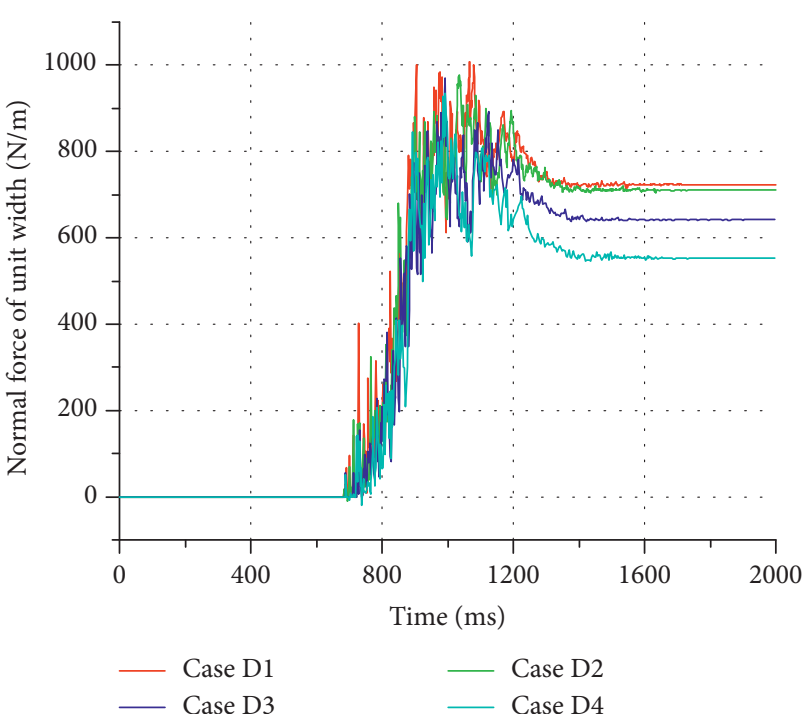

(a)

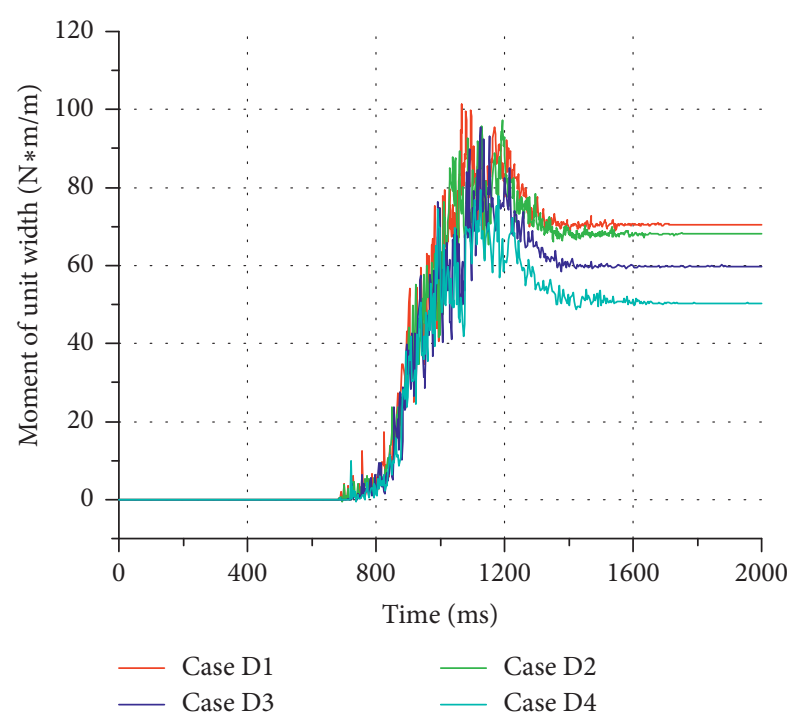

(b)

FIGURE 18: Time history of the impact of the graded granular flow on RNB with different mesh sizes: (a) time history of the unit-width normal force; (b) time history of the unit-width moment.

such as collisions and friction, which is called grain-size segregation $[46,47]$. To analyze the characteristics of grainsize segregation in front of the RNB, the grain distributions under different mesh size conditions were compared, as shown in Figure 19.

As seen from the distribution diagram shown in Figure 19, the graded granular flow stopped and accumulated under the obstruction of the RNB and showed a certain grain size distribution characteristic. When the mesh size was relatively large, the grain size gradually decreased along the flume in the upstream direction due to the passing-through phenomenon, while the distribution along the height of the RNB exhibited more large grains in the upper part and more small grains in the lower part. The reason for this grain size distribution may be that the larger grains had more chance to be subjected to the upward impact force during the collision, and the small grains were more likely to be deposited through intergranular pore spaces to the bottom. The movement and accumulation processes of graded grains are complicated. At present, the understanding of the mechanism of grain-size segregation is not deep enough, so it still needs constant study $[48,49]$. The grain size distribution determines the partitioning of the physicomechanical properties of the deposits in front of the RNB including the density, porosity, and permeability, which is the premise for the study of the forces on the RNB and for the stability analysis and cleanup of deposits. The discrete element method can effectively simulate the obstruction process of an RNB toward a complex graded granular flow, thus providing a reliable reference for the optimal design of RNB structures.

In actual engineering, natural grains usually have a wide gradation distribution, and it is almost inevitable that fine grains pass through the RNB; thus, it is not necessary to obstruct all grains. When a sufficiently small mesh size is set, the force chain formed between the grains can still effectively obstruct most of the grains. At the same time, graded grains with more complex flow characteristics than those of uniform-size grains have a strong fluctuating impact on the RNB through more frequent collisions and form a special grain size distribution in front of the RNB. The discrete element numerical simulation can capture this information and provide a reference for reasonable RNB setting to achieve the expected protection effect.

\section{Discussion}

RNB is a common engineering structure that can effectively obstruct granular flow disasters. Since it is difficult to obtain information such as the granular flow velocity and the time history of the forces acting on an RNB during the obstruction process using existing measurement technology, numerical simulations become an inevitable choice for use in the design and optimization of RNB structures.

In this study, a typical granular flow experiment was numerically simulated. Based on the verification of the correctness of the numerical simulation, the numerical analysis method was used to compare the protective effect of different RNBs on granular flows and the time history of the forces and moments acting on the RNB, and some useful conclusions were obtained. However, the simplified experiments and calculation models still have some ineluctable differences relative to actual protection engineering. For example, an actual RNB may have deformability, and the deformation of the structure will cause a new structural stress distribution. At the same time, whether the material of the mesh structure has sufficient strength and whether the RNB and the boundary have a reliable connection also become primary factors in maintaining the safety of the protective structure. These factors have not been considered in this study, so they are the direction in which the numerical 


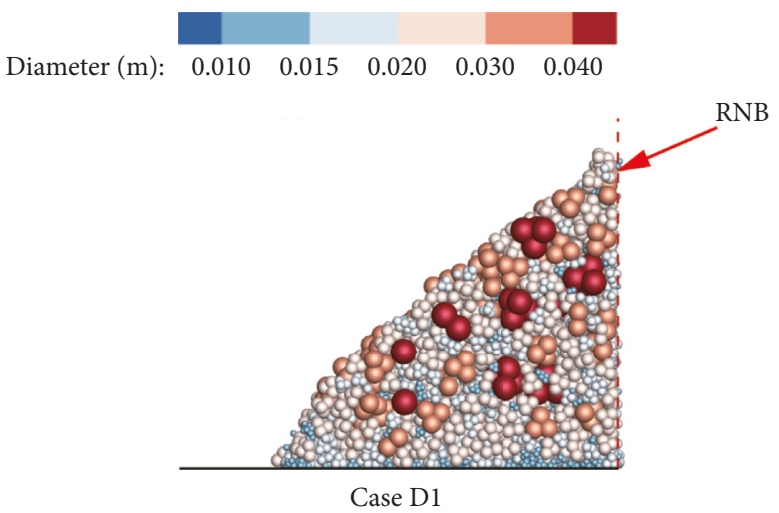

(a)
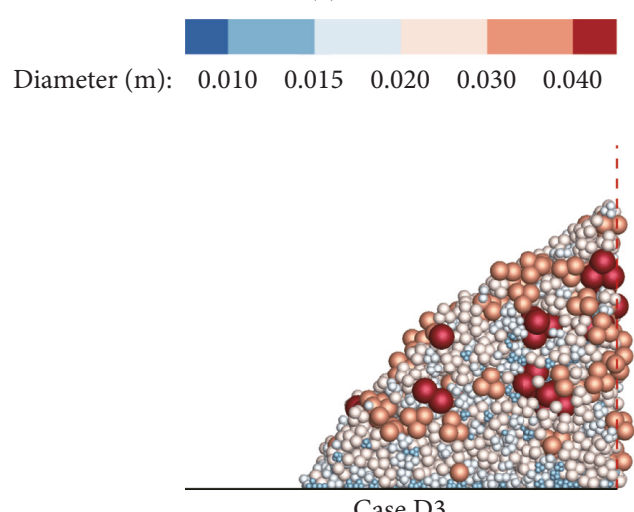

(c)
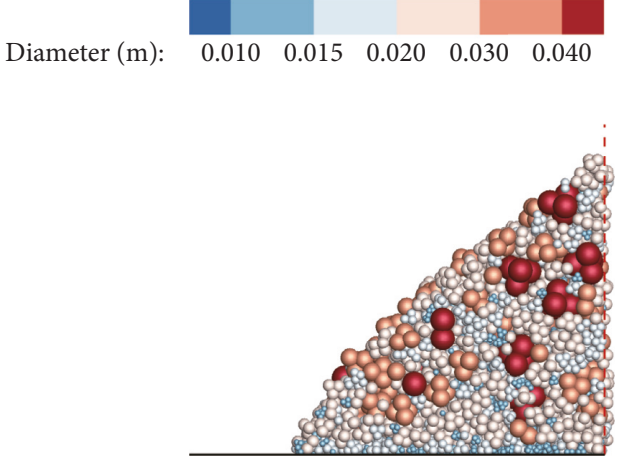

Case D2

(b)
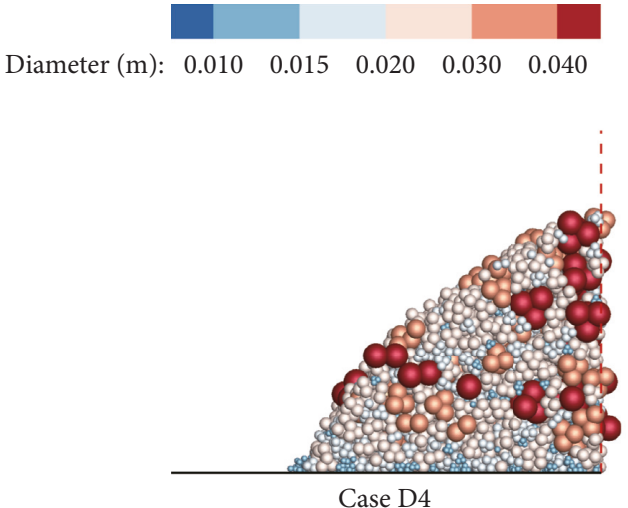

(d)

Figure 19: Distribution diagram of the grain size.

model needs to be further improved and developed. Although the influences of several factors, such as the RNB stiffness and deformation, were neglected in the calculation of the obstruction by the RNB, the results can still show the influence of the structural form and arrangement of the RNB on the main aspects, such as the forces acting on the RNB and the obstruction efficiency toward grains under the condition that the structural rigidity and strength are sufficient. Therefore, these studies apply to RNB with high stiffness and strength. However, if the RNB stiffness is insufficient, or even if the structure is flexible in actual engineering, the conclusions of this study will no longer be valid, and further research must be conducted to dynamically couple the calculations of the RNB and granular flow so that the numerical simulation can fully present the influence of the deformation or even damage of the mesh structure on the dynamic process.

In nature, grains have a wide gradation distribution, so the phenomenon in which fine grains pass through the RNB is almost inevitable in the prevention of actual disasters. At this point, combined with the grading characteristics of the protected rock and soil, the granular flow can be obstructed by setting multiple layers of RNB. As this method helps achieve grading obstruction and reduce the forces acting on the single-layer RNB, the failure risk of the single-layer RNB due to excessive force is reduced, thus providing a reliable solution to a complex engineering problem. Due to limited space, this study did not undertake more in-depth optimization analysis for multilayer RNB settings. Nevertheless, the calculations for the single-layer RNB presented in this study can still reflect some fundamental obstruction characteristics of RNB toward graded granular flow. These studies are useful for further designing multilayer RNB and comprehensive protective measures.

Although the optimization analysis of RNB herein is not combined with specific engineering, the numerical simulation can be used to obtain dynamics data that are difficult to acquire using existing measuring equipment during the obstruction process of granular flow, such as the time history of the granular flow velocity, the time history of the unitwidth normal force and moment acting on the RNB, and the size distribution of the graded grains in front of the RNB. Based on these data, it is possible to place an RNB to avoid harmful phenomena such as overflow, withstand the designed impact load, and achieve the desired obstruction efficiency. Therefore, this study can provide a reference for the optimization of RNB structures and layouts in practical engineering.

\section{Conclusions}

With the characteristics of a simple structure and quick construction, the RNB is an excellent and economical form of a protective structure. Based on the correctness 
verification of the numerical simulation, the obstruction process of an RNB toward granular flows under different setting conditions was analyzed in this study.

It was found that there are several points that should be given attention when designing an RNB. (i) The position setting affects the time history of the forces acting on the RNB and the obstruction efficiency. Under the condition under which the source of the granular flow has been determined, the RNB should be placed upstream to improve the obstruction efficiency and reduce the forces on the RNB; otherwise, the RNB should be placed downstream to provide more reliable protection. (ii) Under effective obstruction from an RNB, the granular flow runs up in front of the RNB, so it is necessary to set an RNB high enough to avoid the overflow phenomenon. (iii) When the mesh size of the RNB is larger than the grain size, although the force chain formed between the grains in front of the RNB can support and obstruct fine grains, it is almost inevitable that small grains will pass through the mesh before the reliable force chain is formed, especially for naturally graded grains. Therefore, to obtain the desired protective effect, it is necessary to set a suitable mesh size. (iv) Under the condition of graded grains, the time history of the forces acting on the RNB and the obstruction efficiency are more complicated. Due to the higher density of the graded grains, the RNB withstands a greater force. Through the collision and action of differently sized grains, the time history of the forces acting on the RNB is more fluctuating.

The abovementioned points can provide a reference for RNB design, but more specific optimization designs (such as the RNB position, height, and mesh size) still need to be obtained through numerical simulations and comparative studies. The numerical simulation can provide not only the obstruction efficiency and the granular flow movement but also the maximum granular flow velocity, the time history of the forces acting on the RNB, and the grain size distribution. In the case of limited measurement techniques for details, the numerical simulation has become an important means to study the obstruction process of RNB structures toward granular flow. Although this study did not take into account the more complex influences of RNB deformation and strength, the analysis has fully demonstrated the main characteristics of granular flow movement and its impact on the RNB, and the obtained results are applicable to RNB with sufficient stiffness and strength. The obstruction efficiency, maximum granular flow velocity, and peak and stable forces obtained by numerical calculations are important indicators for use in RNB design. Through numerical simulation and structural optimization, the RNB can be made more reasonable and effective.

\section{Data Availability}

The numerical calculation data used to support the findings of this study are included within the article and supplementary information files. Previously reported experimental data were used to support this study and are available at doi: 10.1007/s00603-012-0293-3. These prior studies are cited at relevant places within the text as references (Jiang and Towhata [45]).

\section{Conflicts of Interest}

The authors declare that there are no conflicts of interest regarding the publication of this paper.

\section{Acknowledgments}

This work was supported by the National Key Research and Development Program of China (Grant nos. 2016YFC0801603 and 2017YFC1503101), the National Natural Science Foundation of China (Grant nos. 41201007 and 51474048), the Fundamental Research Funds for the Central Universities of China (Grant no. N170108029), and the Research Fund for General Science Project of Department of Education of Liaoning Province (Grant no. L2013103).

\section{Supplementary Materials}

The information on calculation data is as follows: The S1.xlsx is data of Figure 5. The S2.xlsx is data of Figure 6. The S3.xlsx is data of Figure 8. The S4.xlsx is data of Figure 9. The S5.xlsx is data of Figure 11. The S6.xlsx is data of Figure 12. The S7.xlsx is data of Figure 14. The S8.xlsx is data of Figure 15. The S9.xlsx is data of Figure 17. The S10.xlsx is data of Figure 18. These supplementary material files including calculation data are stored in the form of an excel file. The name of the sheet in the file indicates the calculation case. The title line of each sheet (the first line) indicates the name of the variable. Based on these descriptions, all the data supporting the manuscript can be easily found. (Supplementary Materials)

\section{References}

[1] J. E. Costa, Physical Geomorphology of Debris Flows: Developments and Applications of Geomorphology, Springer, Berlin, Germany, 1984.

[2] T. Takahashi, Debris Flow, A A Balkema Publishers, Atlanta, GA, USA, 1991.

[3] D. Rickenmann, "Empirical relationships for debris flows," Natural Hazards, vol. 19, no. 1, pp. 47-77, 1999.

[4] D. F. Van Dine, Debris Flow Control Structures for Forest Engineering, Victoria British Columbia, Ministry of Forests, Victoria, BC, Canada, 1996.

[5] X. Yu, X. Chen, W. Zhao, and J. Chen, "The influence of an EPS concrete buffer layer thickness on debris dams impacted by massive stones in the debris flow," Shock and Vibration, vol. 2015, Article ID 463640, 11 pages, 2015.

[6] X. Chen, P. Cui, Y. You, J. Chen, and D. Li, "Engineering measures for debris flow hazard mitigation in the Wenchuan earthquake area," Engineering Geology, vol. 194, pp. 73-85, 2015.

[7] M. Xiong, X. Meng, S. Wang et al., "Effectiveness of debris flow mitigation strategies in mountainous regions," Progress in Physical Geography: Earth and Environment, vol. 40, no. 6, pp. 768-793, 2016.

[8] T. Mizuyama, "Structural countermeasures for debris flow disasters," International Journal of Erosion Control Engineering, vol. 1, no. 2, pp. 38-43, 2008.

[9] R. Brighenti, A. Segalini, and A. M. Ferrero, "Debris flow hazard mitigation: a simplified analytical model for the design 
of flexible barriers," Computers and Geotechnics, vol. 54, pp. 1-15, 2013.

[10] C. E. Choi, C. W. W. Ng, D. Song et al., "Flume investigation of landslide debris resisting baffles," Canadian Geotechnical Journal, vol. 51, no. 5, pp. 540-553, 2013.

[11] C. W. W. Ng, C. E. Choi, D. Song et al., "Physical modeling of baffles influence on landslide debris mobility," Landslides, vol. 12, no. 1, pp. 1-18, 2015.

[12] Y. C. Tai, Y. Wang, J. M. N. T. Gray, and K. Hutter, "Methods of similitude in granular avalanche flows," Advances in ColdRegion Thermal Engineering and Sciences, vol. 533, pp. 415428, 1999.

[13] S. Hauksson, M. Pagliardi, M. Barbolini, and T. Jóhannesson, "Laboratory measurements of impact forces of supercritical granular flow against mast-like obstacles," Cold Regions Science and Technology, vol. 49, no. 1, pp. 54-63, 2007.

[14] A. M. Abdelrazek, I. Kimura, and Y. Shimizu, "Simulation of three-dimensional rapid free-surface granular flow past different types of obstructions using the SPH method," Journal of Glaciology, vol. 62, no. 232, pp. 335-347, 2016.

[15] L. Bugnion, B. W. McArdell, P. Bartelt, and C. Wendeler, "Measurements of hillslope debris flow impact pressure on obstacles," Landslides, vol. 9, no. 2, pp. 179-187, 2012.

[16] M. Hürlimann, D. Rickenmann, and C. Graf, "Field and monitoring data of debris-flow events in the Swiss Alps," Canadian Geotechnical Journal, vol. 40, no. 1, pp. 161-175, 2003.

[17] C. Berger, B. W. McArdell, and F. Schlunegger, "Sediment transfer patterns at the Illgraben catchment, Switzerland: implications for the time scales of debris flow activities," Geomorphology, vol. 125, no. 3, pp. 421-432, 2011.

[18] K. Hu, F. Wei, and Y. Li, "Real-time measurement and preliminary analysis of debris-flow impact force at Jiangjia Ravine, China," Earth Surface Processes and Landforms, vol. 36, no. 9, pp. 1268-1278, 2011.

[19] Y. Cui, C. E. Choi, L. H. D. Liu, and C. W. W. Ng, "Effects of particle size of mono-disperse granular flows impacting a rigid barrier," Natural Hazards, vol. 91, no. 3, pp. 1179-1201, 2018.

[20] K. M. Hákonardóttir, A. J. Hogg, T. Jóhannesson, and G. G. Tómasson, "A laboratory study of the retarding effects of braking mounds on snow avalanches," Journal of Glaciology, vol. 49, no. 165, pp. 191-200, 2003.

[21] J. M. N. T. Gray, Y.-C. Tai, and S. Noelle, "Shock waves, dead zones and particle-free regions in rapid granular free-surface flows," Journal of Fluid Mechanics, vol. 491, pp. 161-181, 2003.

[22] S. Moriguchi, R. I. Borja, A. Yashima, and K. Sawada, "Estimating the impact force generated by granular flow on a rigid obstruction," Acta Geotechnica, vol. 4, no. 1, pp. 57-71, 2009.

[23] W. Ashwood and O. Hungr, "Estimating total resisting force in flexible barrier impacted by a granular avalanche using physical and numerical modeling," Canadian Geotechnical Journal, vol. 53, no. 10, pp. 1700-1717, 2016.

[24] G. Gao and M. A. Meguid, "Modeling the impact of a falling rock cluster on rigid structures," International Journal of Geomechanics, vol. 18, no. 2, article 04017141, 2018.

[25] T. Faug, P. Lachamp, and M. Naaim, "Experimental investigation on steady granular flows interacting with an obstacle down an inclined channel: study of the dead zone upstream from the obstacle. Application to interaction between dense snow avalanches and defence structures," Natural Hazards and Earth System Science, vol. 2, no. 3/4, pp. 187-191, 2002.
[26] D. Mancarella and O. Hungr, "Analysis of run-up of granular avalanches against steep, adverse slopes and protective barriers," Canadian Geotechnical Journal, vol. 47, no. 8, pp. 827-841, 2010.

[27] C. W. W. Ng, C. E. Choi, A. Y. Su, J. S. H. Kwan, and C. Lam, "Large-scale successive boulder impacts on a rigid barrier shielded by gabions," Canadian Geotechnical Journal, vol. 53, no. 10, pp. 1688-1699, 2016.

[28] D. Song, C. W. W. Ng, C. E. Choi, G. G. D. Zhou, J. S. H. Kwan, and R. C. H. Koo, "Influence of debris flow solid fraction on rigid barrier impact," Canadian Geotechnical Journal, vol. 54, no. 10, pp. 1421-1434, 2017.

[29] S. B. Savage and K. Hutter, "The motion of a finite mass of granular material down a rough incline," Journal of Fluid Mechanics, vol. 199, no. -1, pp. 177-215, 1989.

[30] F. Wu, Y. Fan, L. Liang, and C. Wang, "Numerical Simulation of dry granular flow impacting a rigid wall using the discrete element method," PLoS One, vol. 11, no. 8, Article ID e0160756, 2016.

[31] O. Hungr, "A model for the runout analysis of rapid flow slides, debris flows, and avalanches," Canadian Geotechnical Journal, vol. 32, no. 4, pp. 610-623, 1995.

[32] A. Mangeney-Castelnau, J. P. Vilotte, M. O. Bristeau et al., "Numerical modeling of avalanches based on Saint Venant equations using a kinetic scheme," Journal of Geophysical Research Solid Earth, vol. 108, no. B11, article 2527, 2013.

[33] R. M. Iverson and D. L. George, "A depth-averaged debrisflow model that includes the effects of evolving dilatancy. I. Physical basis," Proceedings of The Royal Society A: Mathematical, Physical and Engineering Sciences, vol. 470, no. 2170, article 20130819, 2014.

[34] M. Brunet, L. Moretti, A. Le Friant, A. Mangeney, E. D. Fernández Nieto, and F. Bouchut, "Numerical simulation of the 30-45 ka debris avalanche flow of Montagne Pelée volcano, Martinique: from volcano flank collapse to submarine emplacement," Natural Hazards, vol. 87, no. 2, pp. 1189-1222, 2017.

[35] J.-b. Fei, T.-l. Chen, Y.-x. Jie, B.-y. Zhang, and X.-d. Fu, "A three-dimensional yield-criterion-based flow model for avalanches," Mechanics Research Communications, vol. 73, pp. 25-30, 2016.

[36] J. DeBruyn, "Viewpoint: unifying liquid and granular flow," Physics, vol. 4, article 86, 2011.

[37] Y. Forterre and O. Pouliquen, Granular Flows. Glasses and Grains, Springer, Basel, Switzerland, 2011.

[38] Y.-J. Jiang, Y. Zhao, I. Towhata, and D.-X. Liu, "Influence of particle characteristics on impact event of dry granular flow," Powder Technology, vol. 270, pp. 53-67, 2015.

[39] T. Faug, R. Beguin, and C. Benoit, "Mean steady granular force on a wall over-flowed by free-surface gravity-driven dense flows," Physical Review E, vol. 80, article 021305, 2009.

[40] C. E. Choi, C. W. W. Ng, D. Song, R. P. H. Law, J. S. H. Kwan, and K. K. S. Ho, "A computational investigation of baffle configuration on the impedance of channelized debris flow," Canadian Geotechnical Journal, vol. 52, no. 2, pp. 182-197, 2014.

[41] A. Leonardi, F. K. Wittel, M. Mendoza, R. Vetter, and H. J. Herrmann, "Particle-fluid-structure interaction for debris flow impact on flexible barriers," Computer-Aided Civil and Infrastructure Engineering, vol. 31, no. 5, pp. 323-333, 2014.

[42] R. P. H. Law, C. E. Choi, and C. W. W. Ng, "Discrete element investigation of the influence of debris flow baffles on rigid 
barrier impact," Canadian Geotechnical Journal, vol. 53, no. 2, pp. 179-185, 2015.

[43] M. Jakob and O. Hungr, Debris-Flow Hazards and Related Phenomena, Springer, Berlin, Germany, 2007.

[44] Itasca Consulting Group Inc, PFC Particle Flow Code, Ver. 5.0 Manual, Itasca, Minneapolis, MN, USA, 2014.

[45] Y.-J. Jiang and I. Towhata, "Experimental study of dry granular flow and impact behavior against a rigid retaining wall," Rock Mechanics and Rock Engineering, vol. 46, no. 4, pp. 713-729, 2013.

[46] R. A. Bagnold, "Experiments on a gravity-free dispersion of large solid spheres in a Newtonian fluid under shear," Proceedings of the Royal Society of London. Series A. Mathematical and Physical Sciences, vol. 225, pp. 49-63, 1954.

[47] S. B. Savage and C. K. K. Lun, "Particle size segregation in inclined chute flow of dry cohesionless granular solids," Journal of Fluid Mechanics, vol. 189, no. 189, pp. 311-335, 1988.

[48] W. Zhou, Z. Lai, and G. Ma, "Effect of base roughness on size segregation in dry granular flows," Granular Matter, vol. 18, no. 4, article 83, 2016.

[49] K. van der Vaart, A. R. Thornton, and C. G. Johnson, "Breaking size-segregation waves and mobility feedback in dense granular avalanches," Granular Matter, vol. 20, no. 3, article UNSP46, 2018. 


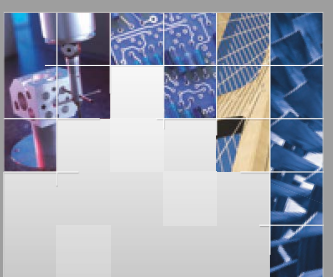

\section{Enfincering}
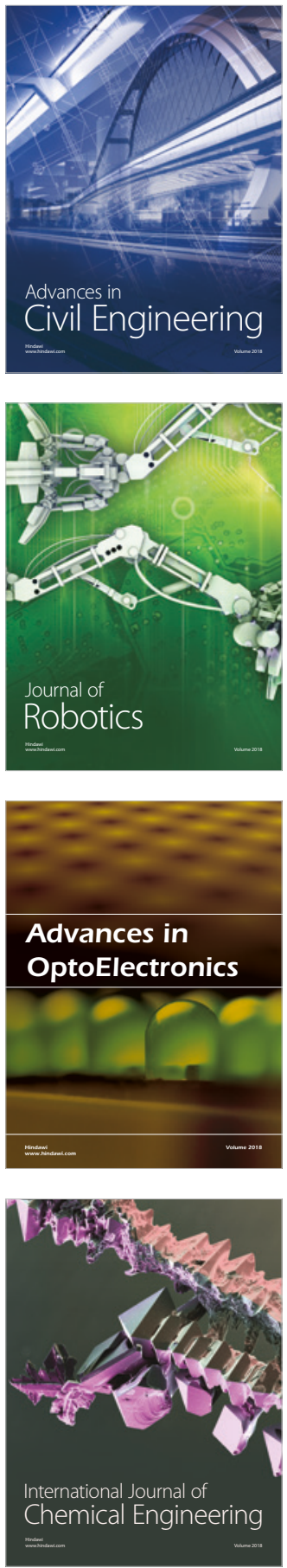

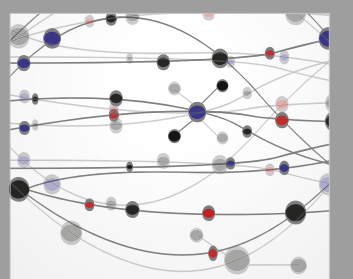

\section{Rotating \\ Machinery}

The Scientific World Journal

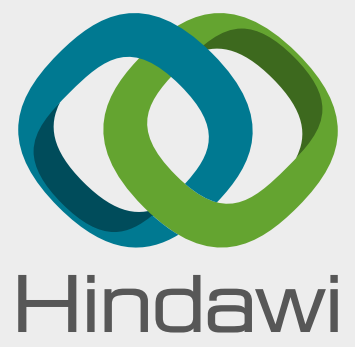

Submit your manuscripts at

www.hindawi.com
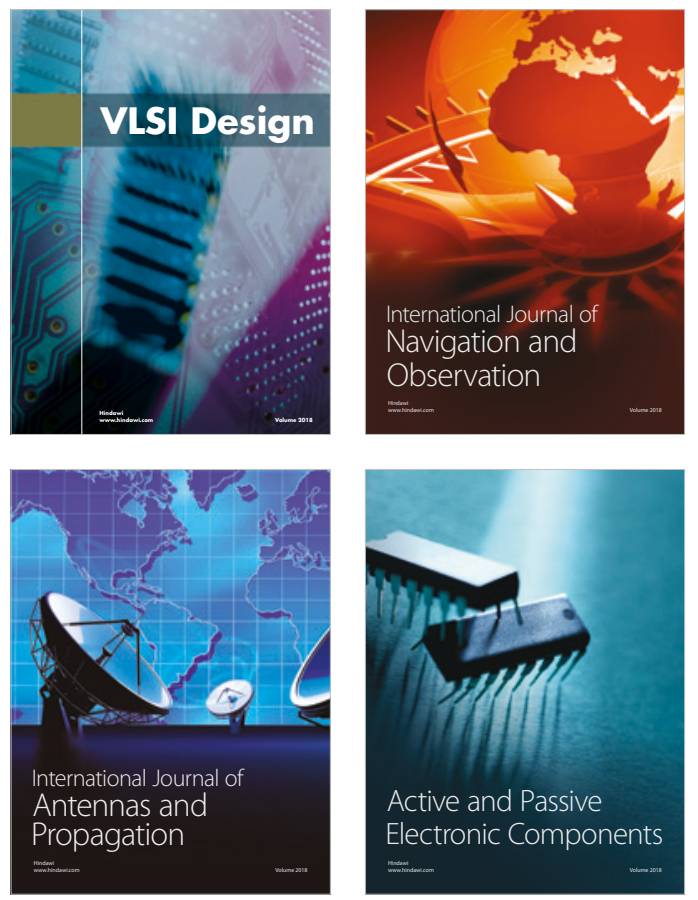
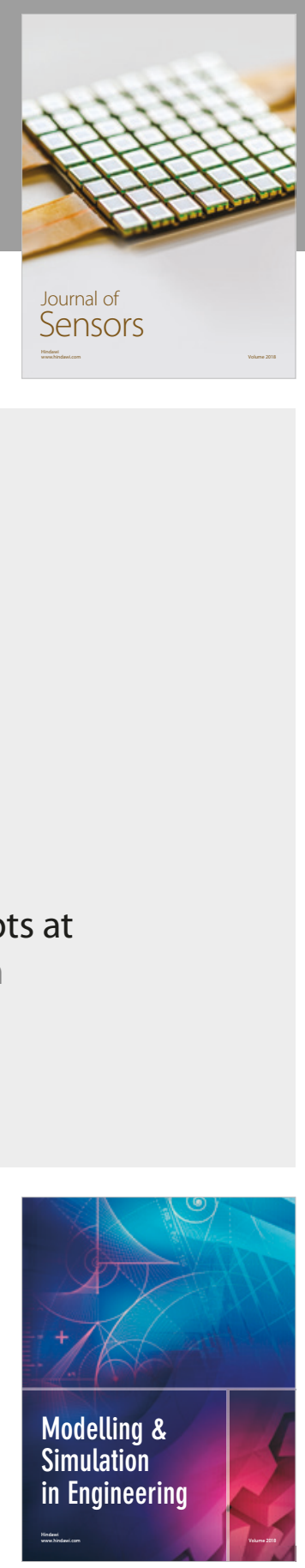

\section{Advances \\ Multimedia}
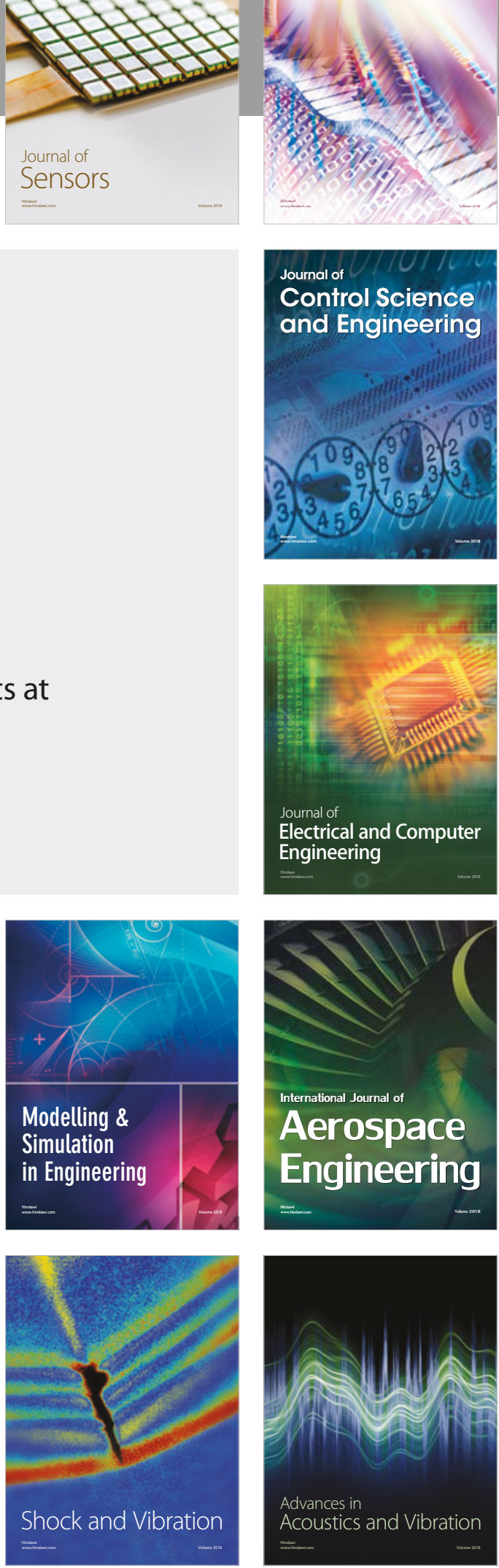